\title{
MODERATELY THICK CIRCULAR PLATES WITH PLANE FACES*
}

\author{
BY
}

H. W. SIBERT

\section{PART I. THeORY}

1. Introduction. Immediately after the propounding of the modern theory of elasticity by Navier in the first quarter of the nineteenth century, a number of mathematicians turned their attention to problems in elasticity. Before long, Poisson and Cauchy had found the differential equations for the displacements of plates which are infinitesimally thin, but it was not until 1883 that a solution for a moderately thick plate was obtained. This solution, found by de Saint-Venant, $\uparrow$ involves rational integral functions of the cylindrical coördinates, $r$ and $z$, where $r$ is measured from the axis of the plate and $z$ from the middle surface. His method consists in finding values for the displacements for several cases of loading of simple type; these solutions are then combined so as to give more complicated loading situations.

In $1887 \mathrm{C}$. Chreef found the solution for a rotating plate. He obtained sets of complementary solutions for the differential equations which must be satisfied by the displacements. With each complementary solution is associated an arbitrary constant; these constants are determined for any particular problem by the loading conditions on the outer surface of the plate.

The problem of a moderately thick circular plate under uniform load was not solved correctly by de Saint-Venant; it was not until after 1900 that the correct solution was obtained by A. E. H. Love. $\S$ Love's solution is an extension of the method developed by J. H. Mitchell in 1900, $\|$ and gives the displacements in terms of rational integral powers of $r$ and $z$.

Subsequent to Love's solution for uniform load, the first important contribution to the literature of moderately thick circular plates was made by A. Nádai $\Upsilon$ in 1920. He obtained, in terms of Bessel functions, the solution for the bending of a moderately thick circular plate under a concentrated load at

\footnotetext{
* Presented to the Society, September 12, 1930; received by the editors in May, 1930.

$\dagger$ Final note of $\S 45$ of his translation of Clebsch, 1883.

$\ddagger$ Transactions of the Cambridge Philosophical Society, vol. 14.

$\S$ Love, The Mathematical Theory of Elasticity, 4th edition, 1927, p. 465.

|| Proceedings of the London Mathematical Society, vol. 31 (1900), p. 100.

If Schweizerische Bauzeitung, vol. 76, No. 22, pp. 257-260.
} 
the center. Several years later, C. A. Garabedian* found correct solutions for a circular plate of constant thickness in terms of polynomials in $r$ and $z$. The next year, A. Timpe $\dagger$ found the same solutions by an entirely different method. In 1926, C. A. Clemmow $\ddagger$ obtained solutions for the bending of a circular plate by adding solutions involving polynomials in $r$ and $z$ to the solution in terms of Bessel functions which had been obtained previously by Nádai.

Both Nádai's and Clemmow's solutions are very cumbersome, even for the case of uniform load. The extension of either of these methods to more complicated types of loading would be almost an impossibility on account of the extremely difficult computations which would arise. The methods used by de Saint-Venant, Love, and Timpe could be extended to more complicated types of loading without giving rise to very difficult computations; but all three of these methods have the disadvantage that, for each new type of loading, the problem must be solved from the very beginning by the process of "trial and error." Garabedian's method is much less involved than those of de Saint-Venant, Love, or Timpe; and, in addition, it gives the necessary machinery for solving any type of loading by means of a single set of equations.

Garabedian's method is based on the assumption that the displacements can be expanded in rational integral powers of a parameter, an assumption which had previously been used by G. D. Birkhoff $\S$ in an attempt to solve problems in circular plates by the use of the calculus of variations.

Although Garabedian was the first to solve successfully problems in moderately thick circular plates by a method involving the assumption that the displacements can be expanded in convergent series, he was not the first to make use of such a postulation. Before 1827, Cauchy\| had made use of this hypothesis; he had solved problems by assuming that the stresses and displacements could be expressed as convergent series in ascending powers of z. But Cauchy concerned himself only with plates which were infinitesimally thin, and neglected all powers of $z$ higher than the second. In 1877, M.Lévy, $\mathbb{T}$ in his study of a thick circular plate having no load on either base, made the assumption that the displacements could be expanded in ascending powers of $z$. He did not attempt to find the solution for any given loading condition, but he was able to prove that the displacements could not contain powers of $z$

\footnotetext{
* These Transactions, vol. 25 (1923), pp. 343-398.

$\dagger$ Zeitschrift für Angewandte Mathematik und Mechanik, vol. 4 (1924), pp. 361-376.

$\ddagger$ Proceedings of the Royal Society, London, (A), vol. 112 (1926), pp. 559-598.

$\S$ Philosophical Magazine, London, Edinburgh, and Dublin, (6), vol. 43 (1922).

|| Cauchy's Exercices de Mathématiques, vol. II, pp. 330-348, 1827.

I Liouville's Journal, (3), vol. 3 (1877), p. 219.
} 
greater than the third when the cylinder is weightless and its bases are free from load.

Garabedian, at the close of his paper, sketched a physical argument to show that his series were convergent. Subsequent to the work done on this paper, Garabedian has found the general term of his series, and has been able to establish convergence for a certain class of loading functions. Moreover, he has found connected with his series an infinite set of constants which turn out to be the same set exhibited in this paper.

The present paper was inspired directly by the above-mentioned paper by Garabedian, and hence, indirectly, by the work done by Birkhoff. Although Garabedian's method and the method used in this paper lead to the same results for any given loading condition, they are quite distinct. On the other hand, it should be said of the two methods that, precisely because of the difference in approach, each method sheds light on the other. Indeed, the two methods, in a sense, complement each other and eventually completely clarify a problem which has waited a full century for solution.

The method of solution employed in this paper is based on the assumption that the components of displacement can be developed in positive integral powers of $z$. In $\S 9$ this assumption will be justified by proving that the series defining the displacements are convergent for, a certain class of loading functions.

The nature of the problem makes the employment of cylindrical coördinates desirable. The axis of the plate is taken as the $z$-axis and the middle plane of the plate as the plane $z=0$. Let the upper and lower faces be $z=h$ and $z=-h$, respectively; thus the thickness of the plate is $2 h$. The plate is taken to be homogeneous, isotropic, and only slightly bent; moreover, the plate must be thin enough so that de Saint-Venant's principle of the elastic equivalence of statically equipollent systems of load can be used at the edge (cf. §4). For the sake of simplicity, all stresses and displacements will be assumed to be independent of $\theta$; the advantage of this assumption is that the differential equations which determine the coefficients of $z$ will be ordinary instead of partial.

Love's notation $†$ with some slight modifications will be used. To obtain results in compact form, the star operator introduced by Garabedian (loc. cit.) will be employed; this operator is defined as follows:

$$
A^{*}=\frac{1}{r} \frac{\partial(r A)}{\partial r}=A^{\prime}+\frac{A}{r} .
$$

$\dagger$ In this paper, all references to Love are to the fourth edition of his Mathematical Theory of Elasticity, 1927. 
Although this method is applicable to problems involving displacements in the direction of $\theta$, lack of space makes it desirable to develop the theory only for the case of displacements in the directions of $r$ and $z$. Moreover, only those problems in which the surface tractions are known and the displacements are to be found will be considered. In this type of problem the displacements must satisfy

(1) the stress equations of motion throughout the body,

(2) the surface traction conditions on the upper and lower faces,

(3) the boundary conditions at the edge.

These three requirements, in the order just indicated, will now be discussed.

2. Stress equations of motion. Let $U$ and $w$ denote the displacements in the directions of $r$ and $z$, respectively. The stress equations of motion may be written in the form (Love, pp. 56, 75, 78, 90, 102)

$$
\frac{\partial^{2} U}{\partial z^{2}}+\frac{1}{1-2 \sigma} \frac{\partial w^{\prime}}{\partial z}+\frac{2(1-\sigma)}{1-2 \sigma} U^{* \prime}=\frac{2(1+\sigma)}{E} \rho\left(f_{r}-F_{r}\right),
$$

(1b) $\frac{\partial^{2} w}{\partial z^{2}}+\frac{1}{2(1-\sigma)} \frac{\partial U^{*}}{\partial z}+\frac{1-2 \sigma}{2(1-\sigma)} w^{\prime *}=\frac{(1+\sigma)(1-2 \sigma)}{(1-\sigma) E} \rho\left(f_{z}-F_{z}\right)$,

in which, as usual, $\rho, \sigma$, and $E$ represent, respectively, density, Poisson's ratio, and the modulus of elasticity; while $f$ and $F$ are the acceleration and body force, respectively. All important and familiar applications involving accelerations or body forces will he provided for if it is assumed that $\rho\left(f_{r}-F_{r}\right)$ is proportional to $r$ and $\rho\left(f_{z}-F_{z}\right)$ is a constant. Thus

$$
\begin{aligned}
& \rho\left(f_{r}-F_{r}\right)=c_{r} r, \\
& \rho\left(f_{z}-F_{z}\right)=c_{z}
\end{aligned}
$$

where $c_{r}$ and $c_{z}$ are constants. The quantities $c_{r} r$ and $c_{z}$ will be called the radial and axial mass forces, respectively. Observe that these mass forces may be due to accelerations or to body forces.

3. Surface-traction conditions. The surface tractions applied to the upper and lower faces can be resolved into radial, tangential, and normal components. The positive direction of the normal component will be taken for both the upper and lower faces to be that of the outward drawn normal (Love, p. 75). The positive direction of the radial component will be taken outward on the upper face. It will be taken inward on the lower face, since the axes for the radial, tangential, and normal components must form either a right-handed or a left-handed system on both faces, and it is desirable that the axes for the tangential component should point in the same direction for both faces. The radial and normal components of the surface tractions will 
be designated by $J_{1}$ and $L_{1}$ on the upper face and by $J_{2}$ and $L_{2}$ on the lower face. Note that $L_{1}$ and $L_{2}$ are tensions when positive and pressures when negative.

In order to satisfy the surface-traction conditions on a face, it is necessary that the components of the internal stress at every point of the face should be equal to the corresponding component of the surface traction at that point. Since the displacements in the direction of $\theta$ are not being considered in this paper, the tangential components of the surface tractions and of the stress will be taken to be zero. Thus the surface-traction conditions on the two faces are

$$
\begin{aligned}
& \left.\widehat{z z}\right|_{z=h}=L_{1},\left.\quad \widehat{z z}\right|_{z=-h}=L_{2} ; \\
& \left.\widehat{r z}\right|_{z=h}=J_{1},\left.\quad \widehat{r z}\right|_{z=-h}=J_{2} .
\end{aligned}
$$

4. Boundary conditions at an edge. In applying boundary conditions at an edge, it is important to distinguish two cases:

(a) the stresses or displacements may be assigned values for every $z$ in the interval from $z=-h$ to $z=h$;

(b) the displacements may have prescribed values at only a limited number of points, or values may be assigned to certain resultant stresses and to certain resultant stress moments taken along a vertical element of an edge.

In the first case, the solution obtained is exact; moreover, this solution applies to a plate of any thickness and may be called a three-dimensional solution. In the second case, the solution is mathematically rigorous, but is only approximate in the physical sense unless for every value of $z$ at the edge the surface tractions are precisely in agreement with the corresponding internal stresses as calculated from the values obtained for the displacements. This type of solution, when not exact, is essentially two-dimensional in character, since this type requires that the thickness of the plate be small as compared with the diameter.

The two-dimensional type of solution is the one used in this paper. The resultant stresses and the resultant stress moments mentioned in case (b) above are (Love, p. 465)

$$
\begin{aligned}
T_{r} & =\int_{-h}^{h} \widehat{r r} d z, \\
N_{r} & =\int_{-h}^{h} \widehat{z r} d z, \\
G_{r} & =\int_{-h}^{h} \widehat{r r} z d z .
\end{aligned}
$$


For moderately thick plates, de Saint-Venant's principle states that the actual distribution of the tractions applied to an edge is of no practical importance. Therefore, instead of dealing with the tractions themselves, their force and couple resultants estimated per unit length of the edge-line are considered. Let the components of these resultants be $T, N, G$ in the sense previously assigned to $T_{r}, N_{r}, G_{r}$. It is necessary that the applied tractions be statically equivalent to the stress resultants and the resultant stress moments at the edge; hence, so far as the stresses are concerned, the boundary conditions at an edge are given by the equations

$$
T_{r}=T, N_{r}=N, G_{r}=G .
$$

It is true that the solutions obtained by means of these equations may not be exact, but, if not exact, they will sufficiently approximate the exact solutions for all points which are not too close to the edge of the plate (Love, pp. 131, 132).

5. The $U$ and $w$ systems. It is now possible to determine formally the values of $U$ and $w$ which satisfy equations (1), (2), (4). It is sonvenient to postpone for the present the consideration of mass forces. Thus the right hand members of formulas (1a) and (1b) are set equal to zero.

The fundamental idea underlying this method is the assumption that $U$ and $w$ can be expanded in powers of $z$; that is,

$$
U=\sum_{n=0}^{\infty} U_{n} \frac{z^{n}}{n !}, \quad w=\sum_{n=0}^{\infty} w_{n} \frac{z^{n}}{n !},
$$

in which $U_{n}$ and $w_{n}$ are functions of $r$ only. Substitute (5) in formulas (1), and equate to zero the coefficients of like powers of $z$. The result is

$$
\begin{aligned}
& U_{n}=-\frac{1}{1-2 \sigma}\left\{w_{n-1}^{\prime}+2(1-\sigma) U_{n-2}^{* \prime}\right\}, \\
& w_{n}=-\frac{1}{2(1-\sigma)}\left\{U_{n-1}^{*}+(1-2 \sigma) w_{n-2}^{\prime *}\right\} .
\end{aligned}
$$

By successive applications of the recurrence relations (6a) and (6b), it is possible to express $U_{n}$ and $w_{n}$ directly in terms of $U_{0}, U_{1}, w_{0}, w_{1}$. A material simplification of these formulas is obtained by the introduction of two new functions defined as follows:

$$
\bar{U}_{0}^{*}=\frac{U_{0}^{*}+w_{1}}{1-2 \sigma}, \quad \bar{w}_{0}^{\prime}=\frac{U_{1}-w_{0}^{\prime}}{2(1-\sigma)} .
$$


The final results are

$$
\begin{aligned}
U_{2 i} & =(-1)^{i}\left\{i \bar{U}_{0}+U_{0}\right\}^{\left(*^{\prime}\right)}, \\
U_{2 i+1} & =(-1)^{i}\left\{(i+2-2 \sigma) \bar{w}_{0}+w_{0}\right\}^{\prime(* \prime)^{i}}, \\
w_{2 i} & =(-1)^{i}\left\{i \bar{w}_{0}+w_{0}\right\}^{(\prime *)^{i}}, \\
w_{2 i+1} & =-(-1)^{i}\left\{(i-1+2 \sigma) \bar{U}_{0}+U_{0}\right\}{ }^{*(*)^{i}} .
\end{aligned}
$$

The substitution of (8) in (5) results in the following formulas for $U$ and $w$ :

$$
\begin{aligned}
U= & \sum_{i=0}^{\infty}(-1)^{i}\left\{i \bar{U}_{0}+U_{0}\right\}^{(* \prime)^{i}} \frac{z^{2 i}}{(2 i) !} \\
& +\sum_{i=0}^{\infty}(-1)^{i}\left\{(i+2-2 \sigma)^{w_{0}}+w_{0}\right\}^{\prime(* \prime)} \frac{z^{2 i+1}}{(2 i+1) !}, \\
w= & \sum_{i=0}^{\infty}(-1)^{i}\left\{i \bar{w}_{0}+w_{0}\right\}^{(\prime *)} \frac{z^{2 i}}{(2 i) !} \\
& -\sum_{i=0}^{\infty}(-1)^{i}\left\{(i-1+2 \sigma) \bar{U}_{0}+U_{0}\right\} *(\prime *) \frac{z^{2 i+1}}{(2 i+1) !} .
\end{aligned}
$$

These expressions for the displacements satisfy formally the stress equations of motion, (1a) and (1b), when $U$ and $w$ are infinite series. It can be shown also that they satisfy (1a) and (1b) when $U$ and $w$ are finite series; in this connection, it is natural to seek necessary and sufficient conditions for $U$ and $w$ to terminate.

Consider first the expression for $U$. If $U$ is to have a finite number of terms, both $\left\{i \bar{U}_{0}+U_{0}\right\}^{(* \prime)^{i}}$ and $\left\{(i+2-2 \sigma) \bar{w}_{0}+w_{0}\right\}^{\prime(* \prime)^{i}}$ must eventually vanish and, moreover, independently of each other, since the former is associated with even powers of $z$ and the latter with odd powers of $z$. For $\left\{i \bar{U}_{0}+U_{0}\right\}^{(* \prime)^{i}}$ to vanish eventually, there must exist a smallest integer $\alpha$ for which $\left\{i \bar{U}_{0}+U_{0}\right\}^{(* \prime)^{i}}=0, i \geqq \alpha$.

Let the anti-prime-star operation be defined as that operation which must be performed upon $P^{* \prime}$ in order to change it to $P$. If this operation is performed upon $\left\{\alpha \bar{U}_{0}+U_{0}\right\}^{(* \prime)^{\alpha}}$, it turns out that $\left\{\alpha \bar{U}_{0}+U_{0}\right\}^{(* \prime)^{\alpha-1}}$ must be of the form $\left\{C_{1} r+C_{2} / r\right\}$, where $C_{1}$ and $C_{2}$ are constants. Moreover, since $\alpha$ is a constant, it follows that both $\bar{U}_{0}^{(* \prime)^{\alpha-1}}$ and $U_{0}^{(* \prime \prime)}$ must also be of this form. By performing alternately integrations and inverse-star operations, it may be shown that $U_{0}$ can contain no terms which are not of the form $\left\{\mathrm{Cr}^{2 m-3}\right.$ $\left.+K r^{2 n-1} \log r\right\}$ and that $\bar{U}_{0}^{*}$ can contain no terms which are not of the form $\left\{C^{\prime} r^{2 p-2}+K^{\prime} r^{2 q-2} \log r\right\}, m, n, p, q$ being any positive integers. By a similar argument, it may be demonstrated that $\bar{w}_{0}^{\prime}$ and $w_{0}$ must have the form speci- 
fied above for $U_{0}$ and for $\bar{U}_{0}^{*}$, respectively. Furthermore, it is not difficult to show that the above restrictions on $U_{0}, \bar{U}_{0}^{*}, w_{0}, \bar{w}_{0}^{\prime}$ constitute not only a sufficient condition for $U$ to terminate but also a necessary and sufficient condition for $w$ to have a finite number of terms. Hence it is evident that if $U$ terminate, so also will $w$; and vice versa.

It is now possible to show that $U$ and $w$ satisfy the stress equations of motion when $U$ and $w$ are finite series. The work is the same as for the case when $U$ and $w$ are infinite series except that now the upper limits for each summation must be determined. All of these limits are readily found, since the operation by which a term becomes eventually zero is differentiation or the star operation according as the term has the form $C r^{m}$ or $K r^{n} \log r$, respectively.

It is now necessary to impose upon $U$ and $w$ the requirement that they satisfy the surface-traction conditions on the upper and lower faces. By means of formulas (9) the stresses $\widehat{z z}$ and $\widehat{r z}$ may be written in the following form (Love, pp. 56, 102):

$$
\begin{aligned}
& \text { (10a) } \widehat{z z}=-\frac{E}{1+\sigma} \sum_{i=0}^{\infty}(-1)^{i}\left\{(i-1+\sigma) \bar{U}_{0}+U_{0}\right\} *\left(\prime^{*}\right) \frac{z^{2 i}}{(2 i) !} \\
& -\frac{E}{1+\sigma} \sum_{i=0}^{\infty}(-1)^{i}\left\{(i+1-\sigma) \bar{w}_{0}+w_{0}\right\}^{\left({ }^{\prime *}\right)}{ }^{i+1} \frac{z^{2 i+1}}{(2 i+1) !}, \\
& \text { (10b) } \widehat{r z}=\frac{E}{1+\sigma} \sum_{i=0}^{\infty}(-1)^{i}\left\{(i+1-\sigma) \bar{w}_{0}+w_{0}\right\}^{\prime(* \prime)^{i}} \frac{z^{2 i}}{(2 i) !} \\
& -\frac{E}{1+\sigma} \sum_{i=0}^{\infty}(-1)^{i}\left\{(i+\sigma) \bar{U}_{0}+U_{0}\right\}^{(* \prime)}{ }^{i+1} \frac{z^{2 i+1}}{(2 i+1) !}
\end{aligned}
$$

Substitute (10) in (2), and take the sum and difference not only of the two resulting values of $\widehat{z z}$ but also of the resulting values of $\widehat{r z}$. A simplification will be obtained by the introduction of four new quantities defined as follows:

$$
\begin{array}{ll}
L=L_{1}+L_{2}, & l=L_{1}-L_{2} ; \\
J=J_{1}+J_{2}, & j=J_{1}-J_{2} .
\end{array}
$$

The final result is

$$
\begin{aligned}
& \sum_{i=0}^{\infty}(-1)^{i}\left\{(i-1+\sigma) \bar{U}_{0}+U_{0}\right\} *\left(\prime^{*}\right)^{i} \frac{h^{2 i}}{(2 i) !}=-\frac{1+\sigma}{2 E} L, \\
& \sum_{i=0}^{\infty}(-1)^{i}\left\{(i+1-\sigma) \bar{w}_{0}+w_{0}\right\}^{(\prime *)}{ }^{i+1} \frac{h^{2 i}}{(2 i+1) !}=-\frac{1+\sigma}{2 E h} l, \\
& \sum_{i=0}^{\infty}(-1)^{i}\left\{(i+1-\sigma) \bar{w}_{0}+w_{0}\right\}^{\prime(* \prime)^{i}} \frac{h^{2 i}}{(2 i) !}=\frac{1+\sigma}{2 E} J,
\end{aligned}
$$




$$
\sum_{i=0}^{\infty}(-1)^{i}\left\{(i+\sigma) \bar{U}_{0}+U_{0}\right\}^{(* \prime)^{i+1}} \frac{h^{2 i}}{(2 i+1) !}=-\frac{1+\sigma}{2 E h} j .
$$

These four systems of ordinary linear differential equations determine the displacements save for the arbitrary constants of integration; the latter are to be fixed subsequently by the edge conditions $(\$ 12)$.

6. The determination of $\bar{w}_{0}^{\prime}$ and $w_{0}$. Since $\bar{w}_{0}$ does not appear in the formulas for $U$ and $w$, it is necessary to find only $\bar{w}_{0}^{\prime}$ and $w_{0}$. These two functions may be obtained by an indirect process from equations (13) and (14). It turns out that it is necessary to break up $\bar{w}_{0}^{\prime}$ and $w_{0}^{\prime}$ into terms ordered according to the powers of the ratio $h / r$. For this purpose, the following convenient definition is introduced. If $X$ and $Y$ are two polynomials in $r$ which contain the same number of terms, $Y$ is defined to be of the $n$th order of magnitude as compared with $X$ if each term of $Y$ is proportional to $(h / r)^{n}$ times the corresponding term in $X$. It is easy to demonstrate that both $X^{(* \prime)^{n}} h^{2 n}$ and $X^{(\prime *)^{n}} h^{2 n}$ are of the $(2 n)$ th order of magnitude as compared with $X$.

In order to apply the method of this paper, certain assumptions must be made with regard to $\bar{w}_{0}^{\prime}$ and $w_{0}^{\prime}$; namely, that these functions are polynomials in $r$, and that they involve $h$ in such a manner that their terms may be grouped and arranged in ascending order of magnitude. Since only even powers of $h$ enter in (13) and (14), it is clear that only even orders of magnitude need be provided for in the developments for $\bar{w}_{0}^{\prime}$ and $w_{0}^{\prime}$. Thus it is assumed that $\bar{w}_{0}^{\prime}$ and $w_{0}^{\prime}$ may be written in the form

$$
\bar{w}_{0}^{\prime}=\sum_{n=0}^{\infty} \bar{w}_{2 n, 0}, \quad w_{0}^{\prime}=\sum_{n=0}^{\infty} w_{2 n, 0}^{\prime},
$$

where $\bar{w}_{2 n, 0}^{\prime}$ and $w_{2 n, 0}^{\prime}$ are of the (2n)th order of magnitude as compared with either $\bar{w}_{00}^{\prime}$ or $w_{00}^{\prime}$. The only assumption made with reference to the leading terms $\bar{w}_{00}^{\prime}$ and $w_{00}^{\prime}$ is that they include, in the case that $\bar{w}_{0}^{\prime}$ and $w_{0}^{\prime}$ do not vanish identically, the terms of lowest order of magnitude occurring in either development. Furthermore, it is assumed that $\bar{w}_{00}^{\prime * * *}$ and $w_{00}^{\prime * *}$ cannot both be identically zero unless

$$
\bar{w}_{2 n, 0}^{\prime * \prime *} \equiv w_{2 n, 0}^{\prime * *} \equiv 0 \quad(n=0,1,2, \cdots) .
$$

If (16) is substituted in (13) and (14), it follows that

$$
\begin{aligned}
& \text { (17) } \sum_{i=0}^{\infty}(-1)^{i}\left\{(i+1-\sigma) \sum_{n=0}^{\infty} \bar{w}_{2 n, 0}+\sum_{n=0}^{\infty} w_{2 n, 0}\right\}^{(* *)} \frac{h^{2 i}}{(2 i+1) !}=-\frac{1+\sigma}{2 E h} l, \\
& \text { (18) } \sum_{i=0}^{\infty}(-1)^{i}\left\{(i+1-\sigma) \sum_{n=0}^{\infty} \bar{w}_{2 n, 0}+\sum_{n=0}^{\infty} w_{2 n, 0}\right\}^{\prime(* \prime)} \frac{h^{2 i}}{(2 i) !}=\frac{1+\sigma}{2 E} J .
\end{aligned}
$$


By means of equations (17) and (18), it will be possible to solve for $\bar{w}_{2 n, 0}^{\prime * * *}$ and $w_{2 n, 0}^{\prime * *}, n=0,1,2, \cdots$.

In obtaining $\bar{w}_{2 n, 0}^{\prime * / *}$ and $w_{2 n, 0}^{\prime * / *}$, it is convenient to treat the cases of normal load and shearing traction separately; by the principle of superposition, it will be possible to solve more complicated problems by a synthesis of the separate solutions. The case of normal load will be considered first.

Before the two systems of differential equations obtainable from (17) and (18) can be written out, it is necessary to determine the order of magnitude of $\{-(1+\sigma) l /(2 E h)\}$. If this quantity is assumed to be of the same order of magnitude as $\bar{w}_{00}^{\prime *}$ or $w_{00}^{\prime *}$, the two equations of lowest order of magnitude in (17) and (18) are, respectively, $(1-\sigma) \bar{w}_{00}^{\prime *}+w_{00}^{\prime *}=-(1+\sigma) l /(2 E h)$ and $(1-\sigma) \bar{w}_{00}^{\prime}+w_{00}^{\prime}=0$. If each term of the latter is starred, an equation is obtained which is inconsistent with the former; hence $\{-(1+\sigma) l /(2 E h)\}$ cannot be of the same order of magnitude as $\bar{w}_{00}^{\prime *}$ or $w_{00}^{\prime *}$. For the present, it will be assumed that this quantity is of the second order of magnitude as compared with $\bar{w}_{00}^{\prime *}$ or $w_{00}^{\prime *}$.

The two systems of equations which result from equating all terms of the same order of magnitude in (17) and (18) may now be written out. They are, respectively,

$$
\begin{aligned}
& \left\{(1-\sigma) \bar{w}_{00}+w_{00}\right\}^{\prime *}=0 \\
& \left\{(1-\sigma) \bar{w}_{20}+w_{20}\right\}^{\prime *}-\left\{(2-\sigma) \bar{w}_{00}+w_{00}\right\}^{(*)^{2}} \frac{h^{2}}{3 !}=-\frac{1+\sigma}{2 E h} l
\end{aligned}
$$

$$
\begin{aligned}
& \left\{(1-\sigma) \bar{w}_{00}+w_{00}\right\}^{\prime}=0 \\
& \left\{(1-\sigma) \bar{w}_{20}+w_{20}\right\}^{\prime}-\left\{(2-\sigma) \bar{w}_{00}+w_{00}\right\}^{\prime * \prime} \frac{h^{2}}{2 !}=0,
\end{aligned}
$$

It is not possible to obtain $\bar{w}_{00}^{\prime *}$ and $w_{00}^{\prime *}$ directly from (19.0) and (20.0) since the latter equation, when starred, becomes identical with the former. It will be possible, though, to find $\bar{w}_{00}^{\prime * *}$ and $w_{00}^{\prime * *}$; and, this done, $\bar{w}_{00}^{\prime *}$ and $w_{00}^{\prime *}$ may be found by quadratures.

The first step in finding $\bar{w}_{00}^{\prime * *}$ and $w_{00}^{\prime * * *}$ is the formation of a third system of equations by starring $(20.2 n)$ and subtracting from $(19.2 n)$; the equations thus obtained will be in simpler form if each is multiplied by $\left(3 / h^{2}\right)$. The final result is 
(21.0) $0=0$,

(21.2) $\left\{(2-\sigma) \bar{w}_{00}+w_{00}\right\}^{(1 *)^{2}}=-\frac{3(1+\sigma)}{2 E h^{3}} l$,

$$
\left\{(2-\sigma) \bar{w}_{20}+w_{20}\right\}^{(\prime *)^{2}}-\left\{(3-\sigma) \bar{w}_{00}+w_{00}\right\}(* *)^{2} \frac{\sigma \cdot 2 h^{2}}{5 !}=0,
$$

Equations (19) and (21) were obtained on the assumption that $\{-(1+\sigma) l /(2 E h)\}$ was of the second order of magnitude as compared with $\bar{w}_{00}^{\prime *}$ or $w_{00}^{\prime *}$. It has already been proved that this quantity is not of the same order of magnitude as $\bar{w}_{00}^{\prime *}$ or $w_{00}^{\prime *}$; it is now possible to show that it cannot be of order higher than the second. If it were of the fourth or higher order, equation (21.2) would be $\left\{(2-\sigma) \bar{w}_{00}+w_{00}\right\}^{(*)^{2}}=0$. The result of solving this equation simultaneously with either (19.0) or (20.0) is $\bar{w}_{00}^{\prime * *} \equiv w_{00}^{\prime * * *} \equiv 0$. Since $l$ must appear on the right hand side of some one of the equations (21), it is not possible that $\bar{w}_{2 n, 0}^{\prime \prime *} \equiv w_{2 n, 0}^{\prime * *} \equiv 0, n=0,1,2, \cdots$. But by a previous assumption, this implies that $\bar{w}_{00}^{\prime * *}$ and $w_{00}^{\prime \prime *}$ cannot both be identically zero. From this contradiction, it follows that $\{-(1+\sigma) l /(2 E h)\}$ is of the second order of magnitude.

By means of (19), (20), and (21), it will be possible to obtain $\bar{w}_{2 m, 0}^{\prime * * *}$ and $w_{2 n, 0}^{\prime \prime *}$ for $n=0,1,2, \cdots$. The procedure consists in solving $(21.2 n+2)$ simultaneously with (20.2n). Accordingly, the star-prime-star operation is performed upon each equation of (20); the resulting set of equations is designated by $(20)^{* * *}$. In order to secure a notation appropriate to the discussion which follows, (20.0)*** and (21.2) are written in the following form:

$$
\begin{aligned}
& \left\{(1-\sigma) \bar{w}_{00}+w_{00}\right\}^{(/ *)^{2}}=-\frac{3(1+\sigma)}{2 E h^{3}} d_{0} l, \\
& \left\{(2-\sigma) \bar{w}_{00}+w_{00}\right\}^{(/ *)^{2}}=-\frac{3(1+\sigma)}{2 E h^{3}} b_{0} l,
\end{aligned}
$$

where $b_{0}=1, d_{0}=0$. The result of solving these two equations simultaneously is

$$
\begin{aligned}
& \bar{w}_{00}^{\prime * *}=-\frac{3(1+\sigma)}{2 E h^{3}}\left\{b_{0}-d_{0}\right\} l, \\
& w_{00}^{w^{\prime *}}=\frac{3(1+\sigma)}{2 E h^{3}}\left\{(1-\sigma) b_{0}-(2-\sigma) d_{0}\right\} l .
\end{aligned}
$$


If these values of $\bar{w}_{00}^{\prime * *}$ and $w_{00}^{\prime * *}$ are substituted in $(20.2) * / *$ and (21.4), we get

$$
\begin{aligned}
& \left\{(1-\sigma) \bar{w}_{20}+w_{20}\right\}^{(\prime *)^{2}}=-\frac{3(1+\sigma)}{2 E h^{3}} d_{1} h^{2} l^{\prime *}, \\
& \left\{(2-\sigma) \bar{w}_{20}+w_{20}\right\}^{(\prime *)^{2}}=-\frac{3(1+\sigma)}{2 E h^{3}} b_{1} h^{2} l^{\prime *},
\end{aligned}
$$

where

$$
b_{1}=\frac{6 \cdot 2\left(2 \cdot b_{0}-1 \cdot d_{0}\right)}{5 !}, \quad d_{1}=\frac{1 \cdot b_{0}-0 \cdot d_{0}}{2 !}
$$

If this pair of equations is solved simultaneously, the result is

$$
\begin{aligned}
& \bar{w}_{20}^{\prime * *}=-\frac{3(1+\sigma)}{2 E h^{3}}\left\{b_{1}-d_{1}\right\} h^{2} l^{\prime *}, \\
& w_{20}^{\prime * *}=\frac{3(1+\sigma)}{2 E h^{3}}\left\{(1-\sigma) b_{1}-(2-\sigma) d_{1}\right\} h^{2} l^{\prime *} .
\end{aligned}
$$

By continuing this process, the following general formulas are obtained:

$$
\begin{array}{ll}
\bar{w}_{2 n, 0}^{\prime * *}=-\frac{3(1+\sigma)}{2 E h^{3}}\left\{b_{n}-d_{n}\right\} h^{2 n} l_{(\prime *)}^{n} & (n=0,1,2, \cdots), \\
w_{2 n, 0}^{\prime * * *}=\frac{3(1+\sigma)}{2 E h^{3}}\left\{(1-\sigma) b_{n}-(2-\sigma) d_{n}\right\} h^{2 n} l_{(\prime *)}^{n} & (n=0,1,2, \cdots)
\end{array}
$$

where $b_{n}$ and $d_{n}$ are given by the formulas

$$
\begin{aligned}
& b_{n}=6 \sum_{i=0}^{n-1}(-1) \frac{(i+2)\left\{(i+2) b_{n-1-i}-(i+1) d_{n-1-i}\right\}}{(2 i+5) !} \\
& d_{n}=\sum_{i=0}^{n-1}(-1) \frac{(i+1) b_{n-1-i}-i d_{n-1-i}}{(2 i+2) !} \quad(n=1,2,3, \cdots) ;
\end{aligned}
$$

$\dagger \bar{w}_{2 n, 0}^{\prime \prime \prime *}$ and $\dot{w}_{2 n, 0}^{\prime \prime \prime *}$ can also be found by solving equations $(21.2 n+2)$ simultaneously with equations $(19.2 n)$. Again let $b_{0}=1, d_{0}=0$; then, by a process similar to that employed above, the following values for $b_{n}$ and $d_{n}$ may be deduced:

$\left(24^{\prime} \mathrm{dd}\right)$

$$
\begin{aligned}
& b_{n}=6 \sum_{i=0}^{n-1}(-1)^{i} \frac{(i+2)\left\{(i+2) b_{n-1-i}-(i+1) d_{n-1-i}\right\}}{(2 i+5) !} \quad(n=1,2,3, \cdots) ; \\
& d_{n}=\sum_{i=0}^{n-1}(-1)^{i} \frac{(i+1) b_{n-1-i}-i d_{n-1-i}}{(2 i+3) !} \quad(n=2,3,4, \cdots) \text {; } \\
& d_{1}=\frac{1 \cdot b_{0}-0 \cdot d_{0}}{3 !}+\frac{1}{3}=\frac{1}{2} \text {. }
\end{aligned}
$$

It may be proved without difficulty that the above values of $b_{n}$ and $d_{n}$ are precisely those given by formulas (24c) and (24d). 
Star-prime-star (16), and make use of (24); the result is

$$
\begin{aligned}
& \bar{w}_{0}^{\prime * * *}=-\frac{3(1+\sigma)}{2 E h^{3}} \sum_{n=0}^{\infty}\left\{b_{n}-d_{n}\right\} h^{2 n} l^{(* *)^{n}}, \\
& w_{0}^{\prime * * *}=\frac{3(1+\sigma)}{2 E h^{3}} \sum_{n=0}^{\infty}\left\{(1-\sigma) b_{n}-(2-\sigma) d_{n}\right\} h^{2 n} l^{(* *)^{n}} .
\end{aligned}
$$

It is not yet possible to solve for $\bar{w}_{0}^{\prime}$ and $w_{0}$, since these two quantities are not independent of each other. The relation between them is found as follows. Transfer all except the first two terms of equations $(20.2 n)$ to the right hand side; by means of (24a), (24b), and (16), there results

$$
\begin{aligned}
(1-\sigma) \bar{w}_{0}^{\prime}+w_{0}^{\prime}-\left\{(2-\sigma) \bar{w}_{0}^{\prime}+w_{0}\right\}^{\prime *} \frac{h^{2}}{2 !} & \\
= & -\frac{3(1+\sigma)}{2 E h^{3}} \sum_{n=0}^{\infty}\left\{d_{n+1}-\frac{b_{n}}{2}\right\} h^{2 n+2 l^{\prime(*))^{n-1}} .}
\end{aligned}
$$

This equation may be obtained in more convenient form. Star-prime each term, and make use of (24e) and (24f); the result is

$$
\left\{(1-\sigma) \bar{w}_{0}+w_{0}\right\}^{\prime * \prime}=-\frac{3(1+\sigma)}{2 E h^{3}} \sum_{n=0}^{\infty} d_{n} h^{2 n} l^{\prime(* \prime)^{n-1}} .
$$

Substitute in $(24 \mathrm{~g})$ the value of $\bar{w}_{0}^{\prime * \prime}$ obtained from $(24 \mathrm{~h})$; the final result is

$$
\begin{aligned}
(1-\sigma) \bar{w}_{0}^{\prime}+w_{0}^{\prime} & +\frac{h^{2}}{2(1-\sigma)} w_{0}^{\prime * \prime}=-\frac{3(1+\sigma)}{2 E h^{3}} \sum_{n=0}^{\infty} d_{n} h^{2 n} l^{\prime(*)^{\prime n-2}} \\
& +\frac{3(1+\sigma)}{2 E h^{3}} \sum_{n=0}^{\infty} \frac{(1-\sigma) b_{n}-(2-\sigma) d_{n}}{2(1-\sigma)} h^{2 n+2 l^{\prime(* \prime \prime)}}{ }^{n-1}
\end{aligned}
$$

It is now possible to solve for $\bar{w}_{0}^{\prime}$ and $w_{0}$. There are two possible methods of procedure. The simpler of the two is first to find $w_{0}$ from (24f) and then obtain $\bar{w}_{0}^{\prime}$ by means of $(24 \mathrm{i})$. Let $\bar{w}_{0 c}^{\prime}$ and $w_{0 c}$ designate the complementary solutions of $\bar{w}_{0}^{\prime}$ and $w_{0}$, respectively; and let $\bar{w}_{0 p}^{\prime}$ and $w_{0 p}$ be the corresponding particular solutions. The complementary solutions are obtained by solving the homogeneous equations associated with equations (24e), (24f), (24i). From (24f), there results

$$
w_{0 c}=K_{1} r^{2} \log r+K_{2} r^{2}+K_{3} \log r+K_{4},
$$

where $K_{1}, K_{2}, K_{3}, K_{4}$ are arbitrary constants. The substitution in (24i) of the values of $w_{0 c}^{\prime}$ and $w_{0 c}^{\prime * \prime}$ obtained from (24j) results in 
(24k) $\bar{w}_{0 c}=-\frac{1}{1-\sigma}\left[K_{1}\left\{2 r \log r+r+\frac{2 h^{2}}{(1-\sigma) r}\right\}+2 K_{2} r+\frac{K_{3}}{r}\right]$.

It turns out that the complementary solutions for all types of loading are given by $(24 j)$ and (24k).

The particular solutions when $\bar{w}_{0}^{\prime * *}$ and $w_{0}^{\prime * / *}$ are infinite series will now be found; the case when they terminate will be considered in $\$ 10$. Let the anti-star-prime-star-prime operation be designated by the symbol $(*)^{-2}$, and let $l^{(* *)^{n-2}}$ be the result obtained when $l^{(* *)^{n}}$ is anti-star-prime-star-primed without introduction of arbitrary constants. From (24f), there results

$$
w_{0 p}=\frac{3(1+\sigma)}{2 E h^{3}} \sum_{n=0}^{\infty}\left\{(1-\sigma) b_{n}-(2-\sigma) d_{n}\right\} h^{2 n} l^{(1 *)}{ }^{n-2} \text {. }
$$

Substitute the above value of $w_{0 p}$ in (24i); the result is

$$
\bar{w}_{0 p}^{\prime}=-\frac{3(1+\sigma)}{2 E h^{3}} \sum_{n=0}^{\infty}\left\{b_{n}-d_{n}\right\} h^{2 n} l^{\prime(*))^{n-2}} .
$$

It is evident that this value of $\bar{w}_{0 p}^{\prime}$ satisfies (24e).

For the case in which there is shearing traction only, equations (21) are solved simultaneously with (20). If $\bar{b}_{0}=1$ and $\bar{d}_{0}=0$, the following general formulas are obtained:

$$
\begin{aligned}
\bar{b}_{n} & =6 \sum_{i=0}^{n-1}(-1) \frac{(i+2)\left\{(i+2) \bar{b}_{n-1-i}-(i+1) \bar{d}_{n-1-i}\right\}}{(2 i+5) !} \quad(n=1,2,3, \cdots) ; \\
\bar{d}_{n} & =\sum_{i=0}^{n-1}(-1) \frac{(i+1) \bar{b}_{n-1-i}-i \bar{d}_{n-1-i}}{(2 i+2) !} \quad(n=2,3,4, \cdots) ; \\
\bar{d}_{1} & =\frac{1 \cdot \bar{b}_{0}-0 \cdot \bar{d}_{0}}{2 !}-\frac{1}{3}=\frac{1}{6} ; \\
\bar{w}_{0}^{\prime * / *} & =\frac{3(1+\sigma)}{2 E h^{2}} \sum_{n=0}^{\infty}\left\{b_{n}-d_{n}\right\} h^{2 n} J *(+*)^{n} ; \\
w_{0}^{\prime * / *} & =\frac{3(1+\sigma)}{2 E h^{2}} \sum_{n=0}^{\infty}\left\{(1-\sigma) \bar{b}_{n}-(2-\sigma) \bar{d}_{n}\right\} h^{2 n J *(1 *)^{n} ; \dagger}
\end{aligned}
$$

† Observe that $\bar{w}_{2 n, 0}^{\prime * \prime *}$ and $w_{2 n, 0}^{\prime * / *}$ might also be found by solving equations (21) simultaneously with (19). Since $\bar{b}_{0}=1$ and $\bar{d}_{0}=0$, the results are

$$
\begin{array}{ll}
\bar{b}_{n}=6 \sum_{i=0}^{n-1}(-1)^{i} \frac{(i+2)\left\{(i+2) \bar{b}_{n-1-i}-(i+1) \bar{d}_{n-1-i}\right\}}{(2 i+5) !} & (n=1,2,3, \cdots) ; \\
\bar{d}_{n}=\sum_{i=0}^{n-1}(-1)^{i} \frac{(i+1) \bar{b}_{n-1-i}-i \bar{d}_{n-1-i}}{(2 i+3) !} & (n=1,2,3, \cdots) .
\end{array}
$$

It may readily be proved that the above values of $\bar{b}_{n}$ and $\bar{d}_{n}$ are the same as those given by (25c), (25d), (25dd). 
(25i)

$$
\begin{aligned}
(1-\sigma) \bar{w}_{0}^{\prime}+w_{0}^{\prime} & +\frac{h^{2}}{2(1-\sigma)} w_{0}^{\prime * \prime}=-\frac{3(1+\sigma)}{2 E h^{2}} \sum_{n=0}^{\infty} \bar{d}_{n} h^{2 n} J^{(* \prime)^{n-1}} \\
& \left.+\frac{3(1+\sigma)}{2 E h^{2}} \sum_{n=0}^{\infty} \frac{(1-\sigma) \bar{b}_{n}-(2-\sigma) \bar{d}_{n}}{2(1-\sigma)} h^{2 n+2} J^{(* \prime)}\right)^{n}
\end{aligned}
$$

When $\bar{w}_{0}^{\prime * * *}$ and $w_{0}^{\prime * * *}$, as defined by (25e) and (25f), are infinite series, it turns out that

$$
\begin{aligned}
& \text { (25) } \quad w_{0 p}=\frac{3(1+\sigma)}{2 E h^{2}} \sum_{n=0}^{\infty}\left\{(1-\sigma) \bar{b}_{n}-(2-\sigma) \bar{d}_{n}\right\} h^{2 n} J^{*(\prime *)}{ }^{n-2}, \\
& \text { (25m) } \quad \bar{w}_{0 p}^{\prime}=-\frac{3(1+\sigma)}{2 E h^{2}} \sum_{n=0}^{\infty}\left\{\bar{b}_{n}-\bar{d}_{n}\right\} h^{2 n} J^{(* \prime)^{n-1}} .
\end{aligned}
$$

7. The determination of $\bar{U}_{0}^{*}$ and $U_{0}$. The function $\bar{U}_{0}$ does not appear in the formulas for $U$ and $w$; hence, it is necessary to find only $\bar{U}_{0}^{*}$ and $U_{0}$. Equations (12) and (15), when considered simultaneously, will suffice for the determination of $\bar{U}_{0}^{*}$ and $U_{0}$. The procedure is analogous to that already given for $\bar{w}_{0}^{\prime}$ and $w_{0}$; but it is much simpler, since, in the determination of $\bar{U}_{0}^{*}$ and $U_{0}$, it is not necessary to form a third system of equations corresponding to equations (21). For the case of normal load only, the final results are

$$
\begin{aligned}
& a_{n}=\sum_{i=0}^{n-1}(-1)^{i} \frac{(i+2) a_{n-1-i}-(i+1) c_{n-1-i}}{(2 i+3) !} \quad(n=1,2,3, \cdots) ; \\
& c_{n}=\sum_{i=0}^{n-1}(-1)^{i} \frac{(i+1) a_{n-1-i}-i c_{n-1-i}}{(2 i+2) !} \quad(n=1,2,3, \cdots) \text {; } \\
& \text { (26e) } \quad \bar{U}_{0}^{* \prime}=-\frac{1+\sigma}{2 E} \sum_{n=0}^{\infty}\left\{a_{n}-c_{n}\right\} h^{2 n} L^{\prime(* \prime)^{n}} \text {; } \\
& \text { (26f) } \quad \overline{U_{0}^{* \prime}}=-\frac{1+\sigma}{2 E} \sum_{n=0}^{\infty}\left\{(1-\sigma) a_{n}+\sigma c_{n}\right\} h^{2 n} L^{\prime(* \prime)^{n}} \text {; } \\
& -(1-\sigma) \bar{U}_{0}^{*}+U_{0}^{*}=-\frac{1+\sigma}{2 E} \sum_{n=0}^{\infty} c_{n} h^{2 n} L^{(1 *)^{n}} ; \\
& U_{0 c}=C_{1} r+\frac{C_{2}}{r} \\
& \bar{U}_{0 c}^{*}=\frac{2 C_{1}}{1-\sigma} \text {. }
\end{aligned}
$$

The complementary solutions for all other types of loading are also given by 
(26h) and (26i). If $\bar{U}_{0}^{* \prime}$ and $U_{0}^{* \prime}$, as given by (26e) and (26f), are infinite series, the values of $U_{0 p}$ and $\bar{U}_{0 p}^{*}$ are

$$
\begin{aligned}
& U_{0 p}=-\frac{1+\sigma}{2 E} \sum_{n=0}^{\infty}\left\{(1-\sigma) a_{n}+\sigma c_{n}\right\} h^{2 n} L^{\prime(*)}{ }^{n-1}, \\
& \bar{U}_{0 p}^{*}=-\frac{1+\sigma}{2 E} \sum_{n=0}^{\infty}\left\{a_{n}-c_{n}\right\} h^{2 n} L^{(* *)^{n}} .
\end{aligned}
$$

It should be observed that for the case of normal load, $a_{0}=0, c_{0}=1$.

For the case of shearing force only, $\bar{a}_{0}=1, \bar{c}_{0}=0$. The general formulas are

$$
\begin{array}{ll}
\bar{a}_{n}=\sum_{i=0}^{n-1}(-1) \frac{(i+2) \bar{a}_{n-1-i}-(i+1) \bar{c}_{n-1-i}}{(2 i+3) !} & (n=1,2,3, \cdots) \\
\bar{c}_{n}=\sum_{i=0}^{n-1}(-1) i \frac{(i+1) \bar{a}_{n-1-i}-i \bar{c}_{n-1-i}}{(2 i+2) !} & (n=1,2,3, \cdots)
\end{array}
$$

(27e) $\bar{U}_{0}^{* \prime}=-\frac{1+\sigma}{2 E h} \sum_{n=0}^{\infty}\left\{\bar{a}_{n}-\bar{c}_{n}\right\} h^{2 n} j^{(* \prime)^{n}}$;

$$
\begin{aligned}
& U_{0}^{* \prime}=-\frac{1+\sigma}{2 E h} \sum_{n=0}^{\infty}\left\{(1-\sigma) \bar{a}_{n}+\sigma \bar{c}_{n}\right\} h^{2 n} j^{(* \prime)^{n}} ; \\
& -(1-\sigma) \bar{U}_{0}^{*}+U_{0}^{*}=-\frac{1+\sigma}{2 E h} \sum_{n=0}^{\infty} \bar{c}_{n} h^{2 n} j^{*(*)^{n-1}} .
\end{aligned}
$$

When $\bar{U}_{0}^{* \prime}$ and $U_{0}^{* \prime}$, as defined by (27e) and (27f), are infinite series, it turns out that

$$
\begin{aligned}
& U_{0 p}=-\frac{1+\sigma}{2 E h} \sum_{n=0}^{\infty}\left\{(1-\sigma) \bar{a}_{n}+\sigma \bar{c}_{n}\right\} h^{2 n} j^{(*))^{n-1}}, \\
& \bar{U}_{0 p}^{*}=-\frac{1+\sigma}{2 E h} \sum_{n=0}^{\infty}\left\{\bar{a}_{n}-\bar{c}_{n}\right\} h^{2 n} j^{*(* *)^{n-1}} .
\end{aligned}
$$

8. Upper bounds of the constants. Before convergence of the series for $U$ and $w$ can be established, upper bounds must be determined for the eight constants which enter in the formulas for $\bar{U}_{0}^{* \prime}, U_{0}^{* \prime}, \bar{w}_{0}^{\prime * * *}, w_{0}^{\prime * *}$.

Consider first the constants $\bar{a}_{n}$ and $\bar{c}_{n}$. It may be proved that

$$
\left|\bar{a}_{n}\right|<1 / 2^{n},\left|\bar{c}_{n}\right|<(5 / 9) / 2^{n} \quad(n=2,3, \cdots) .
$$

If $\bar{a}_{1}, \bar{a}_{2}, \bar{c}_{1}, \bar{c}_{2}$ are calculated from (27c) and (27d), it turns out that (28a) is true for $n=2$. The proof, therefore, consists in showing that if 
(28b) $\left|\bar{a}_{k-1-i}\right|<\frac{1}{2^{k-1-i}}, \quad\left|\bar{c}_{k-1-i}\right|<\frac{5}{9} \frac{1}{2^{k-1-i}} \quad(i=0,1, \cdots,(k-3))$,

then

$$
\left|\bar{a}_{k}\right|<1 / 2^{k}, \quad\left|\bar{c}_{k}\right|<(5 / 9) / 2^{k} \quad(k=3,4, \cdots) .
$$

This proof is not difficult to carry through for both $\bar{a}_{n}$ and $\bar{c}_{n}$. In the proof for $\bar{c}_{n}$, it is necessary to use the relation

(28d) $\bar{c}_{n}=\sum_{i=0}^{n-2}(-1) \frac{(i+1)\left\{(i+2) \bar{a}_{n-2-i}-(i+1) \bar{c}_{n-2-i}\right\}}{(2 i+4) !}(n=2,3,4, \cdots)$,

a formula readily derived from $(27 \mathrm{c})$ and $(27 \mathrm{~d})$.

In a closely analogous manner, it may be shown that

$$
\begin{array}{ll}
\left|a_{n}\right|<1 / 2^{n},\left|c_{n}\right|<(5 / 9) / 2^{n} & (n=1,2, \cdots) \\
\left|b_{n}\right|<1 / 3^{n},\left|d_{n}\right|<(3 / 4) / 3^{n} & (n=2,3, \cdots) \\
\left|\bar{b}_{n}\right|<1 / 3^{n},\left|\bar{d}_{n}\right|<(3 / 4) / 3^{n} & (n=1,2, \cdots) .
\end{array}
$$

In obtaining the upper bounds for $d_{n}$ and $\bar{d}_{n}$, it is necessary to use formulas $\left(24^{\prime} \mathrm{d}\right)$ and $\left(25^{\prime} \mathrm{d}\right)$, respectively.

The above upper bounds seem to be the strongest that can be found by the method of absolute values. By means of these inequalities it will be shown in the next article that the $U$ and $w$ series are uniformly convergent for a limited class of load functions. It would be desirable to include in this class of load functions all powers of $r$ for which the series do not terminate, but the present inequalities are not strong enough for this purpose. On the other hand, if a few values of the constants are calculated, their absolute values are found to be considerably smaller than the above upper bounds would indicate. This suggests the possibility of securing, by a more refined analysis, the stronger upper bounds desired.

Since in the proof of convergence there is no particular advantage in using stronger upper bounds for the $c$ 's, $b$ 's, and $d$ 's than for the $a$ 's, it will be sufficient, in preparation for the following article, to write

$$
\begin{array}{r}
\left|a_{n}\right|,\left|\bar{a}_{n}\right|,\left|c_{n}\right|,\left|\bar{c}_{n}\right|,\left|b_{n}\right|,\left|\bar{b}_{n}\right|,\left|d_{n}\right|,\left|\bar{d}_{n}\right| \leqq 1 / 2^{n} \\
(n=0,1,2, \cdots) .
\end{array}
$$

From formulas (28a), (28e), (28f), (28g), it is evident that the above relations hold for $n=2,3,4, \cdots$. By calculating the values of these constants for $n=1$ from the original formulas, it turns out that $(28 \mathrm{~h})$ is also valid for $n=0,1$. 
9. Convergence of the $U$ and $w$ series. Let $U_{c}$ and $w_{c}$ be the complementary solutions of $U$ and $w$, respectively, and let $U_{p}$ and $w_{p}$ be the corresponding particular solutions. There is no need to examine $U_{c}$ and $w_{c}$, since the consideration of those cases in which $U$ and $w$ terminate will be postponed until $\$ 10$. For the same reason, only those values of $U_{0 p}, \bar{U}_{0 p}^{*}, w_{0 p}$, $\bar{w}_{0 p}^{\prime}$ which are defined by infinite series will be considered in this article.

First consider $U_{p}$ when the plate is under normal load only. $U_{p}$ is found by substituting $(26 \mathrm{j}),(26 \mathrm{k}),(24 \mathrm{l}),(24 \mathrm{~m})$ in $(9 \mathrm{a})$. It turns out that $U_{p}$ is composed of four iterated series, the first of which is

$$
\left.-\frac{1+\sigma}{2 E} \sum_{i=0}^{\infty}(-1)^{i} i\left[\sum_{n=0}^{\infty}\left\{a_{n}-c_{n}\right\} h^{2 n} L^{\prime(* \prime)}\right]^{n-1}\right]^{\left(*^{\prime}\right)^{i}} \frac{z^{2 i}}{(2 i) !}
$$

Since it will be shown that each of the above series is absolutely convergent for a certain class of load functions, it will be convenient in the discussion which follows to employ the double series rather than the given iterated series.

Let $A_{1}$ be the double series corresponding to (29a), and let $A$ be the series formed from the absolute values of the terms of $A_{1}$. If $(28 \mathrm{~h})$ is employed, the result is

$$
A \leqq \sum_{i=0}^{\infty} \sum_{n=0}^{\infty} \frac{i}{(2 i) !} \frac{2}{2^{n}} h^{2 n}\left|L^{\prime(* \prime)^{n-1+i}}\right| \dot{z}^{2 i}
$$

Since $i /(2 i) ! \leqq 1 / 2^{i}(i=0,1,2, \cdots)$ and $|z| \leqq h,(29 \mathrm{~b})$ becomes

$$
A \leqq 2 \sum_{i=0}^{\infty} \sum_{n=0}^{\infty} \frac{h^{2(n+i)}}{2^{n+i}}\left|L^{\prime(*))^{n-1+i}}\right| .
$$

Let $r_{0}$ be the outer radius of the plate, and let $q, q_{1}, q_{2}$ be constants such that $0<q<q_{1}<q_{2}=r_{0} / h$. Then

$$
A \leqq 2 \sum_{i=0}^{\infty} \sum_{n=0}^{\infty}\left(\frac{r_{0}}{2^{1 / 2} q}\right)^{2(n+i)}\left|L^{\prime(*)^{n-1+i}}\right|\left(\frac{q h}{r_{0}}\right)^{2(n+i)}
$$

Since $0<q h / r_{0}<q_{1} h / r_{0}<1$, the above double series of positive terms will be uniformly convergent if $L$ is such a function of $r$ that a constant $M$ can be found for which

$$
\left(\frac{r_{0}}{2^{1 / 2} q}\right)^{2(n+i)}\left|L^{\prime(* \prime)}{ }^{n-1+i}\right|<M, 0 \leqq r \leqq r_{0},
$$

for all values of $n$ and $i$.

It may be shown that if $L=p J_{0}\left( \pm 2^{1 / 2} q r / r_{0}\right)$, where $p$ is a constant, then (29e) reduces to $\left\{p r_{0} /\left(2^{1 / 2} q\right)\right\} J_{1}\left( \pm 2^{1 / 2} q r / r_{0}\right)$ for all values of $n$ and $i$. Hence a class of Bessel functions have actually been exhibited for which the iterated 
series (29a) is both absolutely and uniformly convergent in the interval $0 \leqq r \leqq r_{0}$.

By a procedure similar to the foregoing, it may be shown that the series defining either $U_{p}$ or $w_{p}$ will be absolutely and uniformly convergent for normal load or shearing traction under hypotheses analogous to $(29 \mathrm{e})$.

In the case of the example just given, it is readily shown that the series $U_{p}$ and $w_{p}$ may be primed, starred, or differentiated with respect to $z$, term by term, as many times as may be desired. Thus examples have now been exhibited in which all the operations that have been hitherto applied formally are seen to be justified.

10. Finite series. In $\S 5$, necessary and sufficient conditions were given for the $U$ and $w$ series to terminate. From formulas $(24 \mathrm{j}),(24 \mathrm{k}),(26 \mathrm{~h}),(26 \mathrm{i})$ it is apparent that the complementary solutions have the forms which ensure terminating series for the displacements. It remains only to determine the admissible forms of particular solutions in the cases of normal load and shearing traction.

When the plate is subjected to normal load or shearing traction, it is easy to show that a condition sufficient to meet the requirements on $U_{0}, \bar{U}_{0}^{*}, w_{0}$, $\bar{w}_{0}^{\prime}$ given in $\S 5$ is that $L$ and $l$ shall contain no terms which are not of the form

$$
C^{\prime} r^{2 p-2}+K^{\prime} r^{2 q-2} \log r
$$

and that $J$ and $j$ shall contain no terms which are not of the form

$$
C r^{2 m-3}+K r^{2 n-1} \log r
$$

$m, n, p, q$ being any positive integers. Moreover, it may be shown by an argument similar to that employed in $\S 5$ that, assuming $U_{0}, \bar{U}_{0}^{*}, w_{0}, \bar{w}_{0}^{\prime}$ given by series which terminate, the above conditions are also necessary; no attempt will be made in this paper to find necessary conditions in the case in which $U_{0}, \bar{U}_{0}^{*}, w_{0}, \bar{w}_{0}^{\prime}$ are defined by infinite series.

In order to conserve space, only the case in which $L, l, J, j$ contain no logarithmic terms will be considered; in addition, the case in which the shear is proportional to $1 / r$ will also be neglected since for this case the particular solutions involve logarithmic terms.

It is first desirable to study the effect of the prime-star operator on powers of $r$. It may readily be shown that

$$
\begin{aligned}
& \left(r^{2 m}\right)^{(* *)^{n}} \neq 0,=0 ; n \leqq m, n>m, \\
& \left(r^{2 m}\right)^{(*)^{n}} \neq 0,=0 ; n<m, n \geqq m,
\end{aligned}
$$

where $m=0,1,2, \cdots$, and $n$ is any integer, or zero. 
No loss in generality will ensue if it is assumed that $L, l, J, j$ contain but one term, since the more complicated types of loading may be considered as the sum of simple loadings which contain one term only. Thus $L$ and $l$ may be written in the form

$$
L=e_{1} r^{2 m}, l=e_{2} r^{2 m} \quad(m=0,1,2, \cdots),
$$

where $e_{1}$ and $e_{2}$ are constants. Substitute (31a) in (26e), (26f), (26g), and make use of (30); the result is

$$
\begin{aligned}
& \bar{U}_{0}^{* \prime}=-\frac{1+\sigma}{2 E} \sum_{n=0}^{m-1}\left\{a_{n}-c_{n}\right\} h^{2 n} L^{\prime(* \prime)^{n}}, \\
& U_{0}^{* \prime}=-\frac{1+\sigma}{2 E} \sum_{n=0}^{m-1}\left\{(1-\sigma) a_{n}+\sigma c_{n}\right\} h^{2 n} L^{\prime(* \prime)^{n}}, \\
& -(1-\sigma) \bar{U}_{0}^{*}+U_{0}^{*}=-\frac{1+\sigma}{2 E} \sum_{n=0}^{m} c_{n} h^{2 n} L^{(* *)^{n}} .
\end{aligned}
$$

$U_{0 p}$ is found by performing the inverse prime-star operation upon (31c); the result is

$$
U_{0 p}=-\frac{1+\sigma}{2 E} \sum_{n=0}^{m}\left\{(1-\sigma) a_{n}+\sigma c_{n}\right\} h^{2 n} L^{\prime(*)}{ }^{n-1} .
$$

It should be observed that $m-1$ could have been used for the upper limit in the summation defining $U_{0 p}$, but the use of $m$ makes it possible to express the subsequent formulas in more concise and elegant form. Substitute (31e) in (31d); the resulting value of $\bar{U}_{0 p}^{*}$ is

$$
\bar{U}_{0 p}^{*}=-\frac{1+\sigma}{2 E} \sum_{n=0}^{m}\left\{a_{n}-c_{n}\right\} h^{2 n} L^{(\prime *)} .
$$

It is evident that this value of $\bar{U}_{0 p}^{*}$ satisfies (31b).

In an analogous manner, $w_{0_{p}}$ and $\bar{w}_{0 p}^{\prime}$ may be written as

$$
\begin{aligned}
& w_{0 p}=\frac{3(1+\sigma)}{2 E h^{3}} \sum_{n=0}^{m+1}\left\{(1-\sigma) b_{n}-(2-\sigma) d_{n}\right\} h^{2 n} l^{(* *)^{n-2}}, \\
& \bar{w}_{0}^{\prime}=-\frac{3(1+\sigma)}{2 E h^{3}} \sum_{n=0}^{m+1}\left\{b_{n}-d_{n}\right\} h^{2 n} l^{\prime(* \prime)}{ }^{n-2}
\end{aligned}
$$

For the case of shear, $J$ and $j$ may be written in the form

$$
J=s_{1} r^{2 m+1}, j=s_{2} r^{2 m+1} \quad(m=0,1,2, \cdots),
$$

where $s_{1}$ and $s_{2}$ are constants. Observe that 


$$
J^{*}=2(m+1) s_{1} r^{2 m}, j^{*}=2(m+1) s_{2} r^{2 m} \quad(m=0,1,2, \cdots) ;
$$

that is, $J^{*}$ and $j^{*}$ are proportional to $r^{2 m}$ and, therefore, formulas (30) may be used to determine the upper limits of $n$. The final results are

$$
\begin{aligned}
& U_{0 p}=-\frac{1+\sigma}{2 E h} \sum_{n=0}^{m+1}\left\{(1-\sigma) \bar{a}_{n}+\sigma \bar{c}_{n}\right\} h^{2 n} j^{(* \prime)}{ }^{n-1}, \\
& \bar{U}_{0 p}^{*}=-\frac{1+\sigma}{2 E h} \sum_{n=0}^{m+1}\left\{\bar{a}_{n}-\bar{c}_{n}\right\} h^{2 n} j^{*(* *)}{ }^{n-1}, \\
& w_{0 p}=\frac{3(1+\sigma)}{2 E h^{2}} \sum_{n=0}^{m+1}\left\{(1-\sigma) \bar{b}_{n}-(2-\sigma) \bar{d}_{n}\right\} h^{2 n} J^{*(\prime *)}{ }^{n-2}, \\
& \bar{w}_{0 p}^{\prime}=-\frac{3(1+\sigma)}{2 E h^{2}} \sum_{n=0}^{m+1}\left\{\bar{b}_{n}-\bar{d}_{n}\right\} h^{2 n} J^{(* \prime)}{ }^{n-1} .
\end{aligned}
$$

The values of $U_{0 p}, \bar{U}_{0 p}^{*}, w_{0 p}, \bar{w}_{0 p}^{\prime}$ for the cases of radial and axial mass forces may be found by a process similar to that employed for the case of normal load. The formulas for radial mass force only are

$$
\begin{aligned}
& U_{0 p}=\frac{1-\sigma^{2}}{8 E} c_{r} r^{3}, \\
& \bar{U}_{0 p}^{*}=\frac{1+\sigma}{2 E} c_{r} r^{2}, \\
& w_{0 p}=\bar{w}_{0 p}^{\prime}=0 .
\end{aligned}
$$

When there is axial mass force only the formulas are

$$
\begin{aligned}
& U_{0 p}=\bar{U}_{0 p}^{*}=0, \\
& w_{0 p}=-\frac{3\left(1-\sigma^{2}\right)}{64 E h^{2}} c_{z} r^{4}, \\
& \bar{w}_{0 p}^{\prime}=\frac{3(1+\sigma)}{16 E h^{2}} c_{z} r^{3}+\frac{3(1+\sigma)}{4(1-\sigma) E} c_{z} r .
\end{aligned}
$$

The complete formulas for the displacements may now be written out. It is convenient to give in full the portion involving the mass forces and to use the symbols $U_{0 p}, \bar{U}_{0 p}^{*}, w_{0 p}, \bar{w}_{0 p}^{\prime}$ for the particular solutions in the cases of normal load and shearing traction. The displacements take the form 
(35a) $U=C_{1} r+\frac{C_{2}}{r}-\left[K_{1}\left\{2 r \log r+r+\frac{4 h^{2}}{(1-\sigma) r}\right\}+2 K_{2} r+\frac{K_{3}}{r}\right] z$

$$
\begin{aligned}
& +\frac{2(2-\sigma)}{3(1-\sigma)} \frac{K_{1}}{r} z^{3}+\frac{1-\sigma^{2}}{8 E} c_{r} r^{3}+\frac{3\left(1-\sigma^{2}\right)}{16 E h^{2}} c_{z} r^{3} z \\
& +\frac{3(1+\sigma)}{2 E} c_{z} r z+\frac{\sigma(1+\sigma)}{2 E} c_{r} r z^{2}-\frac{(2-\sigma)(1+\sigma)}{4 E h^{2}} c_{z} r z^{3} \\
& +\sum_{i=0}(-1)^{i}\left\{i \bar{U}_{0 p}+U_{0 p}\right\}^{(* \prime)^{i}} \frac{z^{2 i}}{(2 i) !} \\
& +\sum_{i=0}(-1)^{i}\left\{(i+2-2 \sigma)^{w_{0 p}}+w_{0 p}\right\}^{\prime(* \prime)^{i}} \frac{z^{2 i+1}}{(2 i+1) !}
\end{aligned}
$$

(35b) $w=K_{1} r^{2} \log r+K_{2} r^{2}+K_{3} \log r+K_{4}-\frac{2 \sigma}{1-\sigma} C_{1} z$

$$
\begin{aligned}
& +\frac{2 \sigma}{1-\sigma}\left\{K_{1}(1+\log r)+K_{2}\right\} z^{2}-\frac{3\left(1-\sigma^{2}\right)}{64 E h^{2}} c_{2} r^{4} \\
& -\frac{\sigma(1+\sigma)}{2 E} c_{r} r^{2} z-\frac{3 \sigma(1+\sigma)}{8 E h^{2}} c_{z} r^{2} z^{2} \\
& -\frac{(1+4 \sigma)(1+\sigma)}{4(1-\sigma) E} c_{z} z^{2}-\frac{\sigma^{2}(1+\sigma)}{3(1-\sigma) E} c_{r} z^{3}+\frac{(1+\sigma)^{2}}{8 E h^{2}} c_{z} z^{4} \\
& +\sum_{i=0}(-1)^{i}\left\{i \bar{w}_{0 p}+w_{0 p}\right\}{ }^{(*)} \frac{i z^{2 i}}{(2 i) !} \\
& -\sum_{i=0}(-1)^{i}\left\{(i-1+2 \sigma) \bar{U}_{0 p}+U_{0 p}\right\} *\left({ }^{\prime *}\right)^{i} \frac{z^{2+1}}{(2 i+1) !} .
\end{aligned}
$$

The upper limits of $i$ in the above summations are, taken in order, the same as the upper limits of $n$ already found for $U_{0 p}, \bar{w}_{0 p}^{\prime}, w_{0 p}, \bar{U}_{0 p}^{*}$, respectively.

It is now possible to express $T_{r}, N_{r}, G_{r}$ in terms of $c_{r}, c_{z}, U_{0 p}, \bar{U}_{0 p}^{*}, w_{0 p}$, $\bar{w}_{0 p}^{\prime}$, and the arbitrary constants involved in (35). At the same time, two additional quantities needed in Part II will be computed, namely,

$$
\begin{aligned}
T_{\theta} & =\int_{-h}^{h} \widehat{\theta \theta} d z, \\
G_{\theta} & =\int_{-h}^{h} \widehat{\theta \theta} z d z .
\end{aligned}
$$


The formulas for $T_{r}$ and $T_{\theta}$ can be simplified by making use of the function $\bar{U}_{0 p}$. This function has hitherto been undefined, since it does not appear in the $U$ and $w$ series. $\bar{U}_{0 p}$ may be assigned any value which is consistent with the formulas already given for $\bar{U}_{0 p}^{*}$; it will be convenient, in each case, to take for the upper limit of $n$ in $\bar{U}_{0 p}$ the value found for $U_{0 p}$. Thus

$$
\begin{aligned}
& \bar{U}_{0 p}=-\frac{1+\sigma}{2 E} \sum_{n=0}^{m}\left\{a_{n}-c_{n}\right\} h^{2 n} L^{\prime(* \prime)}{ }^{n-1}, \\
& \bar{U}_{0 p}=-\frac{1+\sigma}{2 E h} \sum_{n=0}^{m+1}\left\{\bar{a}_{n}-\bar{c}_{n}\right\} h^{2 n} j^{(* \prime)^{n-1}} .
\end{aligned}
$$

By means of (31), (32), and the formulas which define the $a^{\prime}$ s, $b$ 's, $c^{\prime}$ 's, and $d$ 's, it may be shown that the following relations are valid for all cases of normal load and shearing traction:

$$
\begin{aligned}
& \text { (37a) }\left.\sum_{i=0}(-1)^{i}\left\{(i+\sigma) \bar{U}_{0 p}+U_{0 p}\right\}(* \prime)^{i} \frac{h^{2 i+1}}{(2 i+1) !}=-\frac{1+\sigma}{2 E} j^{(* \prime)}\right)^{-1}, \\
& \text { (37b) } \sum_{i=0}(-1)^{i}\left\{(i+1-\sigma) \bar{w}_{0 p}+w_{0 p}\right\}^{\prime(* \prime)} \frac{h^{2 i+1}}{(2 i+1) !}=-\frac{1+\sigma}{2 E} l^{(*)^{-1}}, \\
& \text { (37c) } \sum_{i=0}(-1)^{i}\left\{(i+2-\sigma) \bar{w}_{0 p}+w_{0 p}\right\}^{\prime(* \prime)^{i}} \frac{(i+1) h^{2 i+3}}{(2 i+3) !}=-\frac{1+\sigma}{4 E} h J^{(* \prime)^{-1}} \\
&-\frac{1+\sigma}{4 E} l^{(* *)^{-1}},
\end{aligned}
$$

the upper limits of $i$ in the summations being the same as those for $n$ in $U_{0 p}$, $\bar{w}_{0 p}^{\prime}, \bar{w}_{0 p}^{\prime}$, respectively.

Substitute (35) in the formulas for $\widehat{r r}, \widehat{\theta \theta}, \widehat{r z}$, and put these values of $\widehat{r r}, \widehat{\theta \theta}$, $\widetilde{r z}$ in the formulas for $T_{r}, T_{\theta}, G_{r}, G_{\theta}, N_{r}$, and simplify by means of formulas (37). The final results are

$$
\begin{aligned}
\text { (38a) } \begin{aligned}
T_{r}= & \frac{2 E h}{1-\sigma} C_{1}-\frac{2 E h}{1+\sigma} \frac{C_{2}}{r^{2}}+\frac{3+\sigma}{4} h r^{2} c_{r}+\frac{\sigma(1+\sigma)}{3(1-\sigma)} h^{3} c_{r} \\
& +\frac{2 E \sigma}{1+\sigma} \frac{1}{r} \sum_{i=0}(-1)^{i} \bar{U}_{0 p}^{(* \prime)} \frac{h^{2 i+1}}{(2 i+1) !}-j^{\left(*^{\prime}\right)^{-1},}, \\
\text { (38b) } T_{\theta}= & \frac{2 E h}{1-\sigma} C_{1}+\frac{2 E h}{1+\sigma} \frac{C_{2}}{r^{2}}+\frac{1+3 \sigma}{4} h r^{2} c_{r}+\frac{\sigma(1+\sigma)}{3(1-\sigma)} h^{3} c_{r} \\
& \left.+\frac{2 E \sigma}{1+\sigma} \sum_{i=0}(-1)^{i} \bar{U}_{0 p}^{(* \prime)}\right)^{i} \frac{h^{2 i+1}}{(2 i+1) !}-\frac{1}{r} j^{(* \prime)^{-1}},
\end{aligned}
\end{aligned}
$$


(38c) $G_{r}=-\frac{2 E h^{3}}{3(1-\sigma)}\left\{2 \log r+\frac{3+\sigma}{1+\sigma}-\frac{2(8+\sigma)}{5(1+\sigma)} \frac{h^{2}}{r^{2}}\right\} K_{1}-\frac{4 E h^{3}}{3(1-\sigma)} K_{2}$

$$
\begin{aligned}
& +\frac{2 E h^{3}}{3(1+\sigma)} \frac{K_{3}}{r^{2}}+\left\{\frac{3+\sigma}{8} \frac{r^{2}}{h^{2}}+\frac{24+23 \sigma+3 \sigma^{2}}{30(1-\sigma)}\right\} h^{3} c_{z} \\
& \left.+\frac{4 E \sigma}{1+\sigma} \frac{1}{r} \sum_{i=0}(-1)^{i} \bar{w}_{0 p}^{\prime(* \prime)}\right)^{i} \frac{i+1}{(2 i+3) !} h^{2 i+3}-h J^{(* \prime)}{ }^{-1}, l^{(* \prime *)}{ }^{-1},
\end{aligned}
$$

$$
\begin{aligned}
G_{\theta}= & -\frac{2 E h^{3}}{3(1-\sigma)}\left\{2 \log r+\frac{1+3 \sigma}{1+\sigma}+\frac{2(8+\sigma)}{5(1+\sigma)} \frac{h^{2}}{r^{2}}\right\} K_{1}-\frac{4 E h^{3}}{3(1-\sigma)} K_{2} \\
& -\frac{2 E h^{3}}{3(1+\sigma)} \frac{K_{3}}{r^{2}}+\left\{\frac{1+3 \sigma}{8} \frac{r^{2}}{h^{2}}+\frac{24+23 \sigma+3 \sigma^{2}}{30(1-\sigma)}\right\} h^{3} c_{z} \\
& +\frac{4 E \sigma}{1+\sigma} \sum_{i=0}(-1)^{i} \bar{w}_{0 p}^{(* \prime)} \frac{i}{(2 i+3) !} h^{2 i+3}-\frac{h}{r} J^{(* \prime)-1}-\frac{1}{r} l^{(* / *)-1},
\end{aligned}
$$

(38e) $N_{r}=-\frac{8 E h^{3}}{3\left(1-\sigma^{2}\right)} \frac{K_{1}}{r}+r h c_{z}-l^{(*)}{ }^{-1}$,

where the upper limits of $i$ in the summations are the same as those of $n$ for $\bar{U}_{0 p}, \bar{U}_{0 p}^{\prime}, \bar{w}_{0 p}^{\prime}, \bar{w}_{0 p}^{\prime \prime}$, respectively.

All the formulas necessary for handling a wide class of problems in moderately thick plates have now been obtained. In Part II, the power of this theoretical machinery will be exhibited by applying it to the solution of certain problems of especial interest.

\section{Part II. Application}

11. Introduction to Part II. In applying the theory of Part I, the following problems will be considered:

(i) a complete plate, the load being

(a) a function of $r$ continuous over the whole plate,

(b) a pressure concentrated at the center,

(c) a distribution continuous in each of two concentric zones but discontinuous at their junction-a bizonal problem;

(ii) an incomplete plate, that is, a plate with a concentric hole, the load being

(a) a function of $r$ continuous over the whole plate,

(b) a uniform shear distributed over the inner edge of the plate.

Further types of problems to which this method is applicable will be mentioned at the close of the paper. 
In every problem the surface traction on the faces will be prescribed; hence the displacements will be completely known as soon as the arbitrary constants in (35) have been determined. It turns out that $K_{2}, K_{4}, C_{1}$ depend upon conditions at the outer edge of the plate; $K_{1}, K_{3}, C_{2}$ are determined by conditions at the center or at the inner edge, according as the plate is complete or incomplete.

The outer edge of the plate will always be denoted by $r_{0}$; the radius of an inner edge, or of a junction of two zones, will be called $r_{1}$. In every case, the plate will be fixed in space by demanding that there shall be no axial displacement at the outer edge of the middle surface of the plate; that is

$$
\left.w\right|_{z=0, r=r_{0}}=0 .
$$

If $(39 a)$ is substituted in $(35 b)$, the result is

$$
K_{4}=-K_{1} r_{0}{ }^{2} \log r_{0}-K_{2} r_{0}{ }^{2}-K_{3} \log r_{0}+\frac{3\left(1-\sigma^{2}\right)}{64 E h^{2}} c_{2} r_{0}{ }^{4}-\left.w_{0 p}\right|_{r=r_{0}} .
$$

12. Preliminary formulas for a complete plate. Consider a cylindrical section of radius $r$ cut concentrically from the plate. The sum of the $z$-components of all the external forces acting on this portion of the plate must equal $\rho\left(f_{z}-F_{z}\right)$ times its volume; that is, must equal $2 \pi r^{2} h c_{z}$. Note that the value of $N_{r}$ is constant along the circumference of this section, since all stresses are assumed to be independent of $\theta$. Since the outer normal on both the upper and lower faces was taken as the positive direction for normal load, the following relation is valid for a continuous loading distribution:

$$
2 \pi r N_{r}+\int_{0}^{r} 2 \pi r l d r=2 \pi r^{2} h c_{z}, 0<r \leqq r_{0} .
$$

For a plate whose only load is a pressure of $-W$ pounds concentrated at the center, the corresponding relation is

$$
2 \pi r N_{r}-W=0,0<r \leqq r_{0} .
$$

It is evident that, as a point approaches the axis of the plate, the direction of $\widehat{\theta \theta}$ at that point approaches a radial direction. Since all stresses are independent of $\theta$, it is obvious that any two radial stresses at the same point on the axis of the plate are equal. Hence, it is clear that, for any constant value of $z$,

$$
\lim _{r \rightarrow 0}(\widehat{r r}-\widehat{\theta \theta})=0
$$

Observe that (41a) implies 


$$
\lim _{r \rightarrow 0}\left(T_{r}-T_{\theta}\right)=\lim _{r \rightarrow 0}\left(G_{r}-G_{\theta}\right)=0 .
$$

It should be observed that, although (41a) implies (41b), the converse may not be true. If (41a) is not also satisfied, the solution will not be valid in the immediate neighborhood of the axis of the plate; on the other hand, by de Saint-Venant's principle, such a solution would closely approximate the truth for all points whose distance from the axis is at least equal to the thickness of the plate.

All the formulas necessary for the determination of $K_{1}, K_{3}, K_{4}, C_{2}$ for the case of a complete plate have now been found.

13. Determination of $K_{1}, K_{3}, K_{4}, C_{2}$ for a complete plate with continuous distribution of load. The value of $K_{1}$ will be found first. Substitute in (40a) the value of $l$ given in (31a), and solve for $N_{r}$. The result is

$$
N_{r}=-\frac{e_{2} r^{2 m+1}}{2(m+1)}+r h c_{z}=-l^{(*)^{-1}}+r h c_{z}, 0<r \leqq r_{0} .
$$

The result of substituting this value of $N_{r}$ in (38e) is

$$
K_{1}=0 \text {. }
$$

Observe that (42b) is true for both radial mass force and shearing traction as well as for axial mass force and normal load, since the two former quantities do not appear in either (38e) or (40a).

The values of $C_{2}$ and $K_{3}$ will now be found. Substitute $K_{1}=0$ in (38), and solve for $C_{2}$ and $K_{3}$. The results $(r \neq 0)$ are

$$
\begin{aligned}
& \left.\left(T_{r}-T_{\theta}\right) r^{2}=-\frac{4 E h}{1+\sigma} C_{2}+\frac{1-\sigma}{2} h r c_{r}+r j^{(* \prime)}{ }^{-1}-r^{2} j^{(* \prime)}\right)^{-1} \\
& +\frac{2 E \sigma}{1+\sigma} \sum_{i=0}(-1)^{i}\left\{r \bar{U}_{0 p}^{(* \prime)}-r^{i} \bar{U}_{0 p}^{(* \prime)}{ }^{i},\right\} \frac{h^{2 i+1}}{(2 i+1) !}, \\
& \left.\left(G_{r}-G_{\theta}\right) r^{2}=\frac{4 E h^{3}}{3(1+\sigma)} K_{3}+\frac{1-\sigma}{4} h r^{4} c_{z}+h r J^{(* \prime)}\right)^{-1} \\
& -h r^{2} J^{(* \prime)^{-1}}+r l^{(* *)^{-1}}-r^{2} l^{(* *)^{-1}} \\
& \left.+\frac{4 E \sigma}{1+\sigma} \sum_{i=0}(-1)^{i}\left\{r \bar{w}_{0 p}^{\prime(* \prime)^{i}}-r_{0 p}^{2} \bar{w}_{0 p}^{\prime(*)}\right)^{i}\right\} \frac{i+1}{(2 i+3) !} h^{2 i+3} .
\end{aligned}
$$

It is not difficult to show that $r^{4}$ is the lowest power of $r$ appearing in the right hand member of either (43a) or (43b). Let $r \rightarrow 0$, and make use of (41b). The result is

$$
C_{2}=K_{3}=0 .
$$


If the values of $K_{1}=K_{3}=C_{2}=0$ are substituted in the formulas for $\widehat{r}$ and $\widehat{\theta \theta}$, it turns out that (41a) is satisfied for each of the loading conditions under consideration. Hence, for these loading conditions, the solution will be valid in the neighborhood of the axis of the plate.

The value of $K_{4}$ may now be found in terms of $K_{2}$. Substitute $K_{1}=K_{3}=0$ in (39b). The resulting value of $K_{4}$ is

$$
K_{4}=-K_{2} r_{0}^{2}+\frac{3\left(1-\sigma^{2}\right)}{64 E h^{2}} c_{2} r_{0}^{4}-\left.w_{0 p}\right|_{r=r_{0}} .
$$

14. Determination of $K_{1}, K_{3}, K_{4}, C_{2}$ for a complete plate with a central load. In this article, the only load acting is a downward pressure of $-W$ pounds concentrated at the center of the upper face; that is,

$$
L=l=0,0<r \leqq r_{0} ; L=l=-\infty, r=0 .
$$

The value of $K_{1}$ will be found first. Since $l^{(*)}{ }^{-1}=0$ for every point except the center, formula $(38 \mathrm{e})$ reduces to

$$
N_{r}=-\frac{8 E h^{3}}{3\left(1-\sigma^{2}\right)} \frac{K_{1}}{r}, \quad 0<r \leqq r_{0} .
$$

The result of substituting this value of $N_{r}$ in $(40 \mathrm{~b})$ is

$$
K_{1}=-\frac{3\left(1-\sigma^{2}\right)}{16 \pi E h^{3}} W
$$

$C_{2}$ and $K_{3}$ may now be found. If (45) is substituted in (31e), (31f $\left.\mathrm{f}^{\prime}\right),(31 \mathrm{~g})$, (31h), it turns out that

$$
U_{0 p}=\bar{U}_{0 p}=w_{0 p}=\bar{w}_{0 p}^{\prime}=0,0<r \leqq r_{0} .
$$

Substitute (46b) and (47a) in (38), and recall that $c_{r}=c_{z}=j \equiv J \equiv 0$. The resulting equations yield for $C_{2}$ and $K_{3}$ the values

$$
\text { (47b) } \begin{aligned}
C_{2}= & -\frac{1+\sigma}{4 E h}\left(T_{r}-T_{\theta}\right) r^{2}, 0<r \leqq r_{0} ; \\
\text { (47c) } K_{3}= & \frac{3(1+\sigma)}{4 E h^{3}}\left(G_{r}-G_{\theta}\right) r^{2} \\
& +\frac{3(1+\sigma) W}{16 \pi E h^{3}}\left\{(-1+\sigma) r^{2}+\frac{2(8+\sigma)}{5} h^{2}\right\}, 0<r \leqq r_{0} .
\end{aligned}
$$

Let $r \rightarrow 0$, and make use of (41b); the final result is 


$$
C_{2}=0, K_{3}=\frac{3(1+\sigma)(8+\sigma)}{40 \pi E h} W . \dagger
$$

It is not difficult to show that (41a) is not satisfied. Hence the solution is not valid in the neighborhood of the axis of the plate. This conclusion can be justified from another standpoint. Observe that $\overparen{z z}$ is infinite at the center of the upper face. It is clear that the stress-equations of motion themselves are not valid at the center of the plate, since they were derived on the assumption that the stress remains within the elastic limit. On the other hand, by de Saint-Venant's principle, the actual stress distribution will be closely approximated by this solution at all points whose distance from the axis exceeds the thickness of the plate.

Substitute in (39b) the values of $K_{1}, K_{3}$, and $\varkappa_{0 p}$ already found. The result is

$$
K_{4}=\frac{3\left(1-\sigma^{2}\right)}{16 \pi E h^{3}} W r_{0}^{2} \log r_{0}-\frac{3(1+\sigma)(8+\sigma)}{40 \pi E h} W \log r_{0}-K_{2} r_{0}{ }^{2} .
$$

15. Pure stretching of a complete or incomplete plate with continuous distribution of load. Pure stretching will be defined as that state of strain in which the middle plane is not bent and the deformed plate is symmetrical in that plane. It is convenient to have a criterion for pure stretching in terms of surface tractions and mass forces alone, since they generally constitute the data in any given problem. It is not difficult to show that, for the loading conditions considered in this paper, a sufficient condition for pure stretching is, for the complete plate,

$$
\left.G_{r}\right|_{r=r_{0}}=c_{2}=l \equiv J \equiv 0
$$

† Love (p. 475) gives, for the constant corresponding to $K_{1}$, the same value as that found for $K_{1}$ in (46b). But he takes the constant corresponding to $K_{3}$ to be zero, since that is the only value of $K_{8}$ which permits $w_{0}$ to remain finite at the center of the plate. Incidentally, this value of $K_{8}$ is the only one which makes $w_{0}^{\prime}$ vanish at $r=0$.

It turns out that Love's formula for $w$ (which may be found by substituting his value of $w_{0}$ in the general formulas given on p. 473) leads to an impossible situation. In spite of a negatively infinite load at the central point of the upper surface, the displacements are positively infinite at every point of the axis of the plate except at the central point of the middle surface. Moreover, since the deflection of the middle surface is finite throughout the plate, we find that all points on the central axis which, before strain, were below the middle surface assume a position above it after strain. Evidently, Love's solution is incorrect; hence $K_{3} \neq 0$ and the displacement at the center of the middle plane is not finite. De Saint-Venant in his "Note du $\$ 45$ " of the translation of Clebsch, using a wholly different method, had previously made essentially the same mistake. In a paper published in the Journal de l'Ecole Polytechnique, cahier 26 (1927), p. 89, Garabedian has shown, by an entirely different argument, that both Love's and de Saint-Venant's solutions are in error, and, in the same paper, he gives for the first time the correct solution for a plate centrally loaded. 
and, for the incomplete plate,

$$
\left.G_{r}\right|_{r=r_{0}}=\left.G_{r}\right|_{r=r_{1}}=\left.N_{r}\right|_{r=r_{1}}=c_{z}=l \equiv J \equiv 0 .
$$

It may readily be shown, for both the complete and incomplete plate, that (49) is a sufficient condition that

$$
K_{1}=K_{2}=K_{3}=c_{2}=l \equiv J \equiv 0 .
$$

The value $K_{4}=0$ is obtained by substituting (49c) in (31g) and (39b). The result of substituting (49c) and this value of $K_{4}$ in (35) is

$$
\begin{aligned}
U=C_{1} r+\frac{C_{2}}{r} & +\frac{1-\sigma^{2}}{8 E} c_{r} r^{3}+\frac{\sigma(1+\sigma)}{2 E} c_{r} r z^{2} \\
& +\sum_{i=0}(-1)^{i}\left\{i \bar{U}_{0 p}+U_{0 p}\right\}^{(* \prime)^{-1}} \frac{z^{2 i}}{(2 i) !}, \\
w= & -\frac{2 \sigma}{1-\sigma} C_{1} z-\frac{\sigma(1+\sigma)}{2 E} c_{r} r^{2} z-\frac{\sigma^{2}(1+\sigma)}{3(1-\sigma) E} c_{r} z^{3} \\
& -\sum_{i=0}(-1)^{i}\left\{(i-1+2 \sigma) \bar{U}_{0 p}+U_{0 p}\right\} *(* *) \frac{z^{2 i+1}}{(2 i+1) !} .
\end{aligned}
$$

In dealing with a complete plate, it should be remembered that $C_{2}=0$. In each of the following problems, it is assumed that the distribution of load is such that either $(49 a)$ or $(49 b)$ is satisfied.

Problem I: Complete plate whose outer edge is free to expand, that is, $\left.T_{r}\right|_{r=r_{0}}=0 . C_{1}$ is readily obtained from (38a); its value is

$$
\begin{aligned}
C_{1}= & -\frac{(3+\sigma)(1-\sigma)}{8 E} r_{0}^{2} c_{r}-\frac{\sigma(1+\sigma)}{6 E} h^{2} c_{r} \\
& +\left[\frac{1-\sigma}{2 E h} j^{(* \prime)^{-1}}-\frac{\sigma(1-\sigma)}{1+\sigma} \frac{1}{r} \sum_{i=0}(-1)^{i} \bar{U}_{0 p}^{(* \prime)} \frac{h^{2 i}}{(2 i+1) !}\right]_{r=r_{0}}
\end{aligned}
$$

If this value of $C_{1}$ and the value $C_{2}=0$ are substituted in (50), the displacements will be given for a complete plate under any type of loading which results in pure stretching. From these formulas may be obtained a number of important solutions, a few of which will now be given.

Case Ia: A complete plate acted upon by a radial acceleration due to the rotation of the plate about the z-axis with an angular velocity of $\omega \mathrm{rad}$./sec. This well known solution (Love, p. 148) is obtained by setting $L_{1} \equiv L_{2} \equiv L \equiv j$ $\equiv 0, c_{r}=-\rho \omega^{2}$.

Case Ib: A complete plate on which the only loads are equal constant tensions of magnitude $p$ on both faces. The well known solution, $U=-\sigma p r / E$, $w=p z / E$, is found by setting $c_{r}=j \equiv 0, L_{1}=L_{2}=p, L=2 p$. 
Case Ic: A complete plate with no load other than equal tensions which are proportional to $r^{2}$; that is, $c_{r}=j \equiv 0, L_{1}=L_{2}=p r^{2}, L=2 p r^{2}$. The final result is

$$
\begin{aligned}
& U=-\frac{\sigma}{4 E} p r\left\{(1-\sigma) r_{0}^{2}+(1+\sigma) r^{2}\right\}+\frac{1-\sigma^{2}}{3 E} p r\left(h^{2}-3 z^{2}\right), \\
& w=\frac{p}{2 E} z\left\{\sigma^{2} r_{0}^{2}+2\left(1-\sigma^{2}\right) r^{2}\right\}-\frac{2 \sigma(1+\sigma)}{3 E} p z\left(h^{2}-z^{2}\right) .
\end{aligned}
$$

Case Id: A complete plate on which the only loads are equal outward shearing tractions proportional to $r$; that is, $c_{r}=L \equiv 0, J_{1}=-J_{2}=p r, j=2 p r$. The resulting displacements are

$$
\begin{aligned}
U= & \frac{1-\sigma}{8 E h} p r\left\{(3+\sigma) r_{0}^{2}-(1+\sigma) r^{2}\right\}-\frac{2+5 \sigma-\sigma^{2}}{6 E} h p r \\
& +\frac{(2-\sigma)(1+\sigma)}{2 E h} p r z^{2}, \\
w= & -\frac{\sigma p}{4 E h} z\left\{(3+\sigma) r_{0}^{2}-2(1+\sigma) r^{2}\right\}+\frac{3+2 \sigma+\sigma^{2}}{3 E} h p z \\
& -\frac{(1+\sigma)^{2}}{3 E h} p z^{3} .
\end{aligned}
$$

It is not difficult to show that only in Case Ib does $\widehat{r} r$ vanish at the edge for all $z$. Hence, for this case only, the solution is valid throughout the entire plate, and the ratio of $h$ to $r_{0}$ need not be small. For the other three cases, the solution is not valid in the neighborhood of the edge, and the ratio of $h$ to $r_{0}$ must not be large.

Problem II: An incomplete plate whose outer and inner edges are free to expand, that is, $\left.T_{r}\right|_{r=r_{0}}=\left.T_{r}\right|_{r=r_{1}}=0$. It would be possible to work out as many cases under this problem as were given under Problem I; it will suffice, however, to consider the first case only. $C_{1}$ and $C_{2}$ are obtained from (38a); the results are

$$
\begin{aligned}
& C_{1}=-\frac{(3+\sigma)(1-\sigma)}{8 E}\left(r_{0}{ }^{2}+r_{1}{ }^{2}\right) c_{r}-\frac{\sigma(1+\sigma)}{6 E} h^{2} c_{r}, \\
& C_{2}=-\frac{(3+\sigma)(1+\sigma)}{8 E} r_{0}{ }^{2} r_{1}{ }^{2} c_{r} .
\end{aligned}
$$

If these values of $C_{1}$ and $C_{2}$ and the values $L_{1} \equiv L_{2} \equiv L \equiv j \equiv 0, c_{r}=-\rho \omega^{2}$ are substituted in (50), the well known solution for a rotating incomplete plate is obtained (Love, p. 148). 
Problem III: A complete plate on which the only loads are equal constant pressures of magnitude $-p$ on both faces; that is, $c_{r}=j \equiv 0, L_{1}=L_{2}=-p$, $L=-2 p$. It turns out that

$$
\begin{gathered}
U=C_{1} r+\frac{\sigma(1+\sigma)}{2 E} p r, w=-\frac{2 \sigma}{1-\sigma} C_{1} z-\frac{1-\sigma^{2}}{E} p z, \\
\widehat{r r}=\frac{E}{1-\sigma} C_{1}-\frac{\sigma}{2} p .
\end{gathered}
$$

Case IIIa. The radius of the outer edge is to remain unchanged for all $z$; that is, $\left.U\right|_{r=r_{0}}=0$. The results are

$$
\begin{aligned}
& C_{1}=-\frac{\sigma(1+\sigma)}{2 E} p, \quad U \equiv 0, w=-\frac{(1-2 \sigma)(1+\sigma)}{(1-\sigma) E} p z, \\
& \overparen{r r}=-\frac{\sigma}{1-\sigma} p .
\end{aligned}
$$

Case IIIb. The thickness of the plate is to remain unchanged, that is,

$$
\left.w\right|_{z= \pm h}=0 \text {. }
$$

It turns out that

$$
\begin{aligned}
& C_{1}=-\frac{(1-\sigma)^{2}(1+\sigma)}{2 E \sigma} p, \quad U=-\frac{(1-2 \sigma)(1+\sigma)}{2 E \sigma} p r, w \equiv 0, \\
& \widehat{r r}=-\frac{p}{2 \sigma} .
\end{aligned}
$$

The formulas in Cases IIIa and IIIb are valid throughout the plate; hence there is no limitation on the ratio of thickness to diameter. A similar remark applies to the problem which follows.

Problem IV: An incomplete plate with no loads other than constant pressures, $-p_{0}$ and $-p_{1}$, at the outer and inner edges, respectively. If the values $c_{r}=j \equiv L_{1} \equiv L_{2} \equiv L \equiv 0$ are substituted in (50) and in the formula for $\widehat{r r}$, the result is

$$
U=C_{1} r+\frac{C_{2}}{r}, \quad w=-\frac{2 \sigma}{1-\sigma} C_{1} z, \widehat{r r}=\frac{E}{1-\sigma} C_{1}-\frac{E}{1+\sigma} \frac{C_{2}}{r} .
$$

$C_{1}$ and $C_{2}$ are obtained by setting

$$
\left.\widehat{r r}\right|_{r=r_{0}}=-b_{0} \text { and }\left.\widehat{r r}\right|_{r=r_{1}}=-p_{1} ;
$$

the results are 


$$
C_{1}=-\frac{1-\sigma}{E} \frac{p_{0} r_{0}^{2}-p_{1} r_{1}^{2}}{r_{0}^{2}-r_{1}^{2}}, C_{2}=-\frac{1+\sigma}{E} \frac{\left(p_{0}-p_{1}\right) r_{0}^{2} r_{1}^{2}}{r_{0}^{2}-r_{1}^{2}} .
$$

Most of the solutions just obtained are well known; only cases Ic and Id seem to be given for the first time. The object in finding anew the known solutions has been to exhibit the power and elegance of a method which brings all these solutions, and many others, together under one uniform method of treatment.

16. The bending of a complete plate. In the literature of elasticity there are only a limited number of problems dealing with the bending of moderately thick circular plates. A single solution has been given for a plate bent by its own weight; the problem of the plate loaded uniformly over one face has been solved for several types of edge conditions; a plate whose faces are subjected to shearing traction seems not to have been considered. Also, certain problems involving central load have been given, but some of these have been in error. It would be interesting to treat axial mass force, uniform load, and central load so as to check practically every solution given heretofore; however, lack of space makes it desirable to consider one type of loading only. There is not much difference in the facility with which solutions for these three loading conditions may be found; the case of axial mass force will be chosen since the formulas involved are slightly shorter than those appearing in the two other cases.

Formulas will be needed for $T_{r}$ and $G_{r}$ as well as for the displacements. These formulas are readily obtained by substituting formulas (33) and the values $K_{1}=K_{3}=C_{2}=c_{r}=L \equiv l \equiv j \equiv J \equiv 0$ in (35) and (38). The results are

$$
\begin{aligned}
U= & C_{1} r-2 K_{2} r z+\frac{3\left(1-\sigma^{2}\right) c_{z}}{16 E h^{2}}\left\{r^{2}+\frac{8 h^{2}}{1-\sigma}\right\} r z \\
& -\frac{(1+\sigma)(2-\sigma)}{4 E h^{2}} c_{z} r z^{3},
\end{aligned}
$$

$$
\begin{aligned}
w= & -K_{2}\left(r_{0}^{2}-r^{2}\right)-\frac{2 \sigma}{1-\sigma} C_{1} z+\frac{2 \sigma}{1-\sigma} K_{2} z^{2}+\frac{3\left(1-\sigma^{2}\right)}{64 E h^{2}}\left(r_{0}^{4}-r^{4}\right) c_{z} \\
& -\frac{3 \sigma(1+\sigma) c_{z}}{8 E h^{2}}\left\{r^{2}+\frac{2(1+4 \sigma) h^{2}}{3 \sigma(1-\sigma)}\right\} z^{2}+\frac{(1+\sigma)^{2}}{8 E h^{2}} c_{z} z^{4}
\end{aligned}
$$

(52c) $T_{r}=\frac{2 E h}{1-\sigma} C_{1}$,

$$
G_{r}=-\frac{4 E h^{3}}{3(1-\sigma)} K_{2}+\left\{\frac{3+\sigma}{8} \frac{r^{2}}{h^{2}}+\frac{24+23 \sigma+3 \sigma^{2}}{30(1-\sigma)} h^{3} c_{2}\right\}
$$


At this point, it will be interesting to check formulas (52a) and (52b) against the only solution heretofore given for a plate bent by its own weight. This solution was found by G. H. Bryan (Love, p. 486). Except for a term in $w$, accounted for by an axial translation of the plate as a whole, Bryan's results may be obtained from (52a) and (52b) by setting $c_{z}=\rho g$, $C_{1}=\rho g(1-\sigma) h /(2 E), K_{2}=\rho g(3+7 \sigma) /(8 E)$. His solution would have to be combined with other simpler solutions before it would correspond to any of the commonly used edge conditions.

When the constants $C_{1}$ and $K_{2}$ have been fixed by the conditions at the outer edge, the displacements will be completely determined. Nine types of conditions at the outer edge will be considered; these will be ordered according to the magnitude of the deflection at the center of the middle plane of the plate. Since the deflection at the center is in the direction of the negative $z$-axis, the central deflection of the middle plane increases as $K_{2}$ increases. In order to conserve space, the physical interpretation of the various edge conditions will not be given. The nine cases follow.

Case $\mathrm{S}$.

$\left.G_{r}\right|_{r=r_{0}}=\left.T_{r}\right|_{r=r_{0}}=0$.

Case M-I. $\quad \partial^{2} w /\left.\partial z^{2}\right|_{r=r_{0}, z=0}=0, \partial w /\left.\partial z\right|_{r=r_{0}, z=0}=0$.

Case M-II. $\left.\quad w\right|_{r=r_{0}, z= \pm h}=0$.

Case M-III. $\quad \partial w /\left.\partial z\right|_{r=r_{0}, z= \pm h}=0$.

Case M-IV. $\quad \partial^{2} w /\left.\partial z^{2}\right|_{r=r_{0}, z= \pm h}=0$.

Case C-I. $\left.\quad U\right|_{r=r_{0}, z=0}=\partial U /\left.\partial z\right|_{r=r_{0}, z=0}=0$.

Case C-II. $\left.\quad U\right|_{r=r_{0}, z= \pm h}=0$.

Case C-III. $\left.\quad U\right|_{r=r_{0}, z=0}=\left.w^{\prime}\right|_{r=r_{0}, z= \pm h}=0$.

Case C-IV. $\left.\quad U\right|_{r=r_{0}, z=0}=\left.w^{\prime}\right|_{r=r_{0}, z=0}=0$.

In each case the value of $C_{1}$ turns out to be zero. The values of $K_{2}$ for the nine cases are, respectively,

$$
\begin{aligned}
& K_{2}=\frac{3\left(1-\sigma^{2}\right)}{32 E h^{2}} c_{z}\left\{\frac{3+\sigma}{1+\sigma} r_{0}^{2}+\frac{4\left(24+23 \sigma+3 \sigma^{2}\right)}{15\left(1-\sigma^{2}\right)} h^{2}\right\}, \\
& K_{2}=\frac{3\left(1-\sigma^{2}\right)}{16 E h^{2}} c_{z}\left\{r_{0}^{2}+\frac{2(1+4 \sigma)}{3 \sigma(1-\sigma)} h^{2}\right\}, \\
& K_{2}=\frac{3\left(1-\sigma^{2}\right)}{16 E h^{2}} c_{z}\left\{r_{0}{ }^{2}+\frac{1+8 \sigma+\sigma^{2}}{3 \sigma(1-\sigma)} h^{2}\right\}, \\
& K_{2}=\frac{3\left(1-\sigma^{2}\right)}{16 E h^{2}} c_{2}\left\{r_{0}^{2}+\frac{2(4+\sigma)}{3(1-\sigma)} h^{2}\right\},
\end{aligned}
$$




$$
\begin{aligned}
& K_{2}=\frac{3\left(1-\sigma^{2}\right)}{16 E h^{2}} c_{z}\left\{r_{0}^{2}-\frac{2\left(2-4 \sigma-3 \sigma^{2}\right)}{3 \sigma(1-\sigma)} h^{2}\right\}, \\
& K_{2}=\frac{3\left(1-\sigma^{2}\right)}{32 E h^{2}} c_{z}\left\{r_{0}^{2}+\frac{8 h^{2}}{1-\sigma}\right\}, \\
& K_{2}=\frac{3\left(1-\sigma^{2}\right)}{32 E h^{2}} c_{z}\left\{r_{0}^{2}+\frac{4(4+\sigma)}{3(1-\sigma)} h^{2}\right\}, \\
& K_{2}=\frac{3\left(1-\sigma^{2}\right)}{32 E h^{2}} c_{z}\left\{r_{0}^{2}+\frac{4 \sigma}{1-\sigma} h^{2}\right\}, \\
& K_{2}=\frac{3\left(1-\sigma^{2}\right)}{32 E h^{2}} c_{z} r_{0}^{2} .
\end{aligned}
$$

Cases S and C-IV are those of classical support and classical clamping, respectively. The thin-plate solutions for Cases M-I, C-I, C-II have been given by C. A. Clemmow (loc. cit.) for a plate having uniform pressure on the upper face. The four remaining cases appear to be new.

Clemmow, in an investigation made upon clamped plates (loc. cit.), attempted to obtain a support as rigid as that of classical clamping by cutting a cylinder and head from a solid piece of metal. His expectation was not realized, since the best agreement with his experimental results was given by Case C-I, even when the ratio of thickness to diameter was small. It thus appears that it is virtually impossible to construct a physical type of clamping which will agree with a set of analytical conditions previously advanced. Hence it becomes necessary to devise new analytical conditions which will approximate the physical situations arising in practice. Herein lies a justification for considering Cases C-I, C-II, C-III. It should be borne in mind, however, that only in exceptional cases is the head of a cylinder an integral part of the cylinder itself. Ordinarily, the head is fastened to the cylinder by means of bolts; and this type of fastening is certainly less rigid than that used by Clemmow. It would appear desirable, therefore, to study also the additional cases, M-I, M-II, M-III, M-IV, intermediate between S and C-I.

17. The bending of an incomplete plate. Two problems only will be considered in this article. In each problem, it will be assumed that (i) the inner edge is free and (ii) the mass force is nil and there is no shearing traction on the faces; that is,

$$
\begin{gathered}
\left.T_{r}\right|_{r=r_{1}}=\left.G_{r}\right|_{r=r_{1}}=0, \\
c_{r}=c_{z}=J_{1} \equiv J_{2} \equiv j \equiv J \equiv 0 .
\end{gathered}
$$


Problem I. The only load is a uniform pressure, $-p$, on the upper face; that is,

$$
\left.N_{r}\right|_{r m r_{1}}=L_{2} \equiv 0, L_{1}=L=l=-p .
$$

Problem II. The only load is a downward shearing force of $-W$ pounds distributed uniformly along the inner edge; that is,

$$
L_{1} \equiv L_{2} \equiv L \equiv l \equiv 0 \text {. }
$$

The value of $K_{1}$ for each problem will be found first. The substitution of $c_{z}=0, l=-p$ in (38e) yields the following result for Problem I:

$$
N_{r}=-\frac{8 E h^{3}}{3\left(1-\sigma^{2}\right)} \frac{K_{1}}{r}+\frac{p r}{2} .
$$

The corresponding result for Problem II is

$$
N_{r}=-\frac{8 E h^{3}}{3\left(1-\sigma^{2}\right)} \frac{K_{1}}{r} \text {. }
$$

Consider the inner annular ring cut out from the plate by a concentric cylindrical surface of radius $r$. The equilibrium of this ring requires that

$$
2 \pi r N_{r}-\pi\left(r^{2}-r_{1}^{2}\right) p=0
$$

for Problem I, and that

$$
2 \pi r N_{r}-W=0
$$

for Problem II. The elimination of $N_{r}$ between (55b) and (55c) yields the following value of $K_{1}$ for Problem I:

$$
K_{1}=\frac{3\left(1-\sigma^{2}\right)}{16 E h^{3}} p r_{1}^{2} .
$$

A corresponding procedure in Problem II yields the value

$$
K_{1}=-\frac{3\left(1-\sigma^{2}\right)}{16 \pi E h^{3}} W
$$

It will be convenient to express $C_{2}$ and $K_{3}$ in terms of $C_{1}$ and $K_{2}$, respectively. To accomplish this, $T_{r}$ and $G_{r}$ must first be computed. For Problem I, the results of substituting $c_{r}=c_{z}=j \equiv J \equiv 0, L=l=-p, K_{1}$ $=3\left(1-\sigma^{2}\right) p r_{1}^{2} /\left(16 E h^{3}\right)$ in (38a) and (38c) are

$$
T_{r}=\frac{2 E h}{1-\sigma} C_{1}-\frac{2 E h}{1+\sigma} \frac{C_{2}}{r^{2}}-\frac{\sigma p h}{2},
$$




$$
\begin{aligned}
G_{r}= & -\frac{4 E h^{3}}{3(1-\sigma)} K_{2}+\frac{2 E h^{3}}{3(1+\sigma)} \frac{K_{3}}{r^{2}}+\frac{3+\sigma}{16} p\left(r^{2}-2 r_{1}^{2}\right) \\
& -\frac{1+\sigma}{4} p r_{1}^{2} \log r+\frac{8+\sigma}{20 r^{2}} h^{2} p r_{1}^{2}-\frac{\sigma p h^{2}}{5}
\end{aligned}
$$

For Problem II, the substitution of

$$
c_{r}=c_{z}=j \equiv J \equiv L \equiv l \equiv 0, K_{1}=-3\left(1-\sigma^{2}\right) W /\left(16 \pi E h^{3}\right)
$$

in (38a) and in (38c) results in

$$
\begin{aligned}
T_{r}= & \frac{2 E h}{1-\sigma} C_{1}-\frac{2 E h}{1+\sigma} \frac{C_{2}}{r^{2}}, \\
G_{r}= & -\frac{4 E h^{3}}{3(1-\sigma)} K_{2}+\frac{2 E h^{3}}{3(1+\sigma)} \frac{K_{3}}{r^{2}} \\
& +\frac{W}{8 \pi}\left\{3+\sigma+2(1+\sigma) \log r-\frac{2(8+\sigma)}{5 r^{2}} h^{2}\right\} .
\end{aligned}
$$

The substitution of (54a) in (55e) and (55f) yields the following results for Problem I:

$$
\begin{aligned}
C_{2}= & \frac{1+\sigma}{1-\sigma} r_{1}{ }^{2} C_{1}-\frac{\sigma(1+\sigma)}{4 E} p r_{1}{ }^{2}, \\
K_{3}= & \frac{2(1+\sigma)}{1-\sigma} r_{1}{ }^{2} K_{2} \\
& +\frac{3(1+\sigma)}{8 E h^{3}} p r_{1}^{4}\left\{\frac{3+\sigma}{4}+(1+\sigma) \log r_{1}-\frac{8-3 \sigma}{5 r_{1}{ }^{2}} h^{2}\right\} .
\end{aligned}
$$

By a similar procedure, the following results are obtained for Problem II:

$$
\text { (56g) } \begin{aligned}
C_{2}= & \frac{1+\sigma}{1-\sigma} r_{1}{ }^{2} C_{1}, \\
\text { (56h) } K_{3}= & \frac{2(1+\sigma)}{1-\sigma} r_{1}{ }^{2} K_{2} \\
& -\frac{3(1+\sigma)}{16 \pi E h^{3}} W r_{1}{ }^{2}\left\{3+\sigma+2(1+\sigma) \log r_{1}-\frac{2(8-\sigma)}{5 r_{1}{ }^{2}} h^{2}\right\} .
\end{aligned}
$$

As soon as $w_{0 p}$ is known, the value of $K_{4}$ may be found in terms of $K_{2}$. The values of $w_{0 p}$ (formula (31g)) for Problems I and II turn out to be, respectively,

$$
\begin{aligned}
& w_{0 p}=-\frac{3(1+\sigma)}{16 E h^{3}} f\left(\frac{1-\sigma}{8} r^{4}-\frac{8-3 \sigma}{5} h^{2} r^{2}\right), \\
& w_{0 p}=0 .
\end{aligned}
$$


Substitute in (39b) the values of $K_{1}, K_{3}, w_{0 p}$ already found; the resulting values of $K_{4}$ for Problems I and II are, respectively,

$$
\begin{aligned}
(55 \mathrm{j}) K_{4}= & -K_{2}\left\{r_{0}^{2}+\frac{2(1+\sigma)}{1-\sigma} r_{1}^{2} \log r_{0}\right\}-\frac{3\left(1-\sigma^{2}\right)}{16 E h^{3}} p r_{1}{ }^{2} r_{0}{ }^{2} \log r_{0} \\
& -\frac{3(1+\sigma)}{8 E h^{3}} p r_{1}^{4}\left\{\frac{3+\sigma}{4}+(1+\sigma) \log r_{1}-\frac{8-3 \sigma}{5 r_{1}{ }^{2}} h^{2}\right\} \log r_{0} \\
& +\frac{3(1+\sigma)}{16 E h^{3}} p\left(\frac{1-\sigma}{8} r_{0}^{4}-\frac{8-3 \sigma}{5} h^{2} r_{0}^{2}\right), \\
(56 \mathrm{j}) K_{4}= & -K_{2}\left\{r_{0}{ }^{2}+\frac{2(1+\sigma)}{1-\sigma} r_{1}^{2} \log r_{0}\right\}+\frac{3\left(1-\sigma^{2}\right)}{16 \pi E h^{3}} W r_{0}{ }^{2} \log r_{0} \\
& +\frac{3(1+\sigma)}{16 \pi E h^{3}} W r_{1}{ }^{2}\left\{3+\sigma+2(1+\sigma) \log r_{1}-\frac{2(8+\sigma)}{5 r_{1}{ }^{2}} h^{2}\right\} \log r_{0} .
\end{aligned}
$$

The values of $K_{2}$ and $C_{1}$ for the nine cases of edge conditions given in the last article may now be found. In order to conserve space, these constants will be given only for the case of classical support. The result of setting $\left.T_{r}\right|_{r=r_{0}}=\left.G_{r}\right|_{r=r_{0}}=0$ is

$$
\begin{aligned}
C_{1}= & \frac{\sigma(1-\sigma)}{4 E} p \\
K_{2}= & \frac{3(1-\sigma)}{4 E h^{3}} p\left\{\frac{3+\sigma}{16}\left(r_{0}^{2}-r_{1}^{2}\right)-\frac{\sigma h^{2}}{5}\right\} \\
& -\frac{3\left(1-\sigma^{2}\right) p r_{1}^{2}}{16 E h^{3}\left(r_{0}^{2}-r_{1}^{2}\right)}\left(r_{0}^{2} \log r_{0}-r_{1}^{2} \log r_{1}\right)
\end{aligned}
$$

for Problem I; the results for Problem II are

$$
\begin{aligned}
& C_{1}=0 \\
& K_{2}=\frac{3(1-\sigma)}{16 \pi E h^{3}} W\left\{\frac{3+\sigma}{2}+(1+\sigma) \frac{r_{0}^{2} \log r_{0}-r_{1}{ }^{2} \log r_{1}}{r_{0}{ }^{2}-r_{1}{ }^{2}}\right\} .
\end{aligned}
$$

It is now possible to write out the displacements in full for both problems. However, lack of space makes it undesirable to do this. The solution for the displacements in Problems I and II were apparently given for the first time by A. Timpe in 1924 (loc. cit.).

It turns out that when $r_{1}=0$ the solution given in Problem I becomes the same as that for a complete plate uniformly loaded. Moreover, the solution given in Problem II reduces to that of a complete plate centrally loaded if $W$ 
is kept constant while $r_{1}$ is set equal to zero. It should be observed, however, that one has no right to assume in advance that the complete plate will be a limiting case of the incomplete plate, since, in the case of a complete plate, the constants $C_{2}$ and $K_{3}$ were determined from the conditions

$$
\lim _{r \rightarrow 0}\left(T_{r}-T_{\theta}\right)=\lim _{r \rightarrow 0}\left(G_{r}-G_{\theta}\right)=0,
$$

while, for the incomplete plate, the constants were fixed by the conditions

$$
\left.T_{r}\right|_{r=r_{1}}=\left.G_{r}\right|_{r=r_{1}}=0 \text {. }
$$

18. The bending of a plate under bizonal distribution of load. Since it would require much space to solve a bizonal problem completely, values will be found for only four of the constants; the eight equations necessary for the solution of the eight remaining constants will be given, but no attempt will be made to solve them. The following discussion is valid for all problems in which the only load is a downward pressure of $-W$ pounds distributed continuously over the inner zone, or distributed uniformly over the junction of the two zones. Let the subscripts $i$ and $e$ indicate the interior and exterior zones, respectively.

The inner zone is a complete plate with continuous distribution of load; hence, from $\S 13, K_{1 i}=K_{3 i}=C_{2 i}=0$. Since there is no load on the outer zone, the value of $N_{\text {re }}$ obtained from (38e) is

$$
N_{r e}=-\frac{8 E h^{3}}{3\left(1-\sigma^{2}\right)} \frac{K_{1 e}}{r} .
$$

Consider a section of the plate having for its outer edge a cylinder of radius $r, r_{1}<r<r_{0}$. The equilibrium of this portion of the plate demands that $2 \pi r N_{r e}-W=0$. The substitution of this value of $N_{r e}$ in (57a) results in

$$
K_{1 e}=-\frac{3\left(1-\sigma^{2}\right)}{16 \pi E h^{3}} W .
$$

For the determination of the eight remaining constants, three equations may be obtained from the boundary conditions at the outer edge. Two of these are found by imposing any one of the nine edge conditions given in $\$ 16$ for the determination of $K_{2}$ and $C_{1}$. A third condition results from the requirement that there shall be no axial displacement at the outer edge of the middle surface; that is,

$$
\left.w_{0 i}\right|_{r=r_{0}}=0 .
$$

The five remaining equations must be found from conditions at the junc- 
tion of the two zones. The principle of the equality of action and reaction demands that

$$
\lim _{r \rightarrow r_{1}} \widehat{r r}_{e}=\lim _{r \rightarrow r_{1}} \widehat{r r}_{i}
$$

and hence that

$$
\lim _{r \rightarrow r_{1}} T_{r e}=\lim _{r \rightarrow r_{1}} T_{r i}, \lim _{r \rightarrow r_{1}} G_{r e}=\lim _{r \rightarrow r_{1}} G_{r i} .
$$

It should be noted that although (58) implies (59), the converse may not be true. If (58) is not satisfied, the solution is not valid in the immediate neighborhood of the junction; however, if (59) is satisfied, the solution, according to de Saint-Venant's principle, will hold for all points not too near the boundary of the two zones. Formula (59) furnishes two of the five equations. A third equation results from the assumption that the middle surface of the plate is continuous at the junction of the two zones; that is,

$$
\left.w_{0 i}\right|_{r=r_{1}}=\left.w_{0 e}\right|_{r=r_{1}} \text {. }
$$

The above, or equivalent, conditions at the junction have been used by each person who has attempted to find the solution for a moderately thick plate under bizonal distribution of load. De Saint-Venant (loc. cit.) demanded, in addition, that $w_{0}^{\prime}$ (the slope of the middle surface) should be continuous at the junction. Garabedian, in correcting de Saint-Venant's solution, ${ }^{*}$ demanded that, at the junction, both $T_{\theta}$ and $G_{\theta}$ should be continuous.

It should be noted that one has no more right, a priori, to demand the continuity of $T_{\theta}$ and $G_{\theta}$ at the junction than one has to demand the continuity of $w_{0}^{\prime}$, since the law of the equality of action and reaction does not apply in the case of $T_{\theta}$ and $G_{\theta}$ when the direction of $\widehat{\theta \theta}$ is tangent to the junction. Some other test must be found for determining which of these assumptions is incorrect. Such a test may be found in the case of the loading situations considered in this article, since each of them reduces to the problem of the complete plate loaded centrally when $r_{1}$ is allowed to approach zero while the total load, $W$, is kept constant. The assumption made by de Saint-Venant results in a solution for central load in agreement with that given by Love. But it has already been shown that Love's solution is incorrect; consequently, the assumption made by de Saint-Venant is also incorrect. On the other hand, Garabedian's assumption leads to the correct solution for central load. Hence Garabedian's assumption is a sufficient condition for obtaining the correct solution for central load if $r_{1}$ is allowed to approach zero. The author

* Journal de l'École Polytechnique, vol. 26 (1927), p. 89. 
has used Garabedian's assumption of the equality of $T_{\theta}$ and $G_{\theta}$ at the junction even though he has not been able to prove its necessity; such a proof would seem to involve a difficult problem in the calculus of variations. The employment of the foregoing assumption gives the two remaining equations necessary for the solution of the eight unknown constants.

19. Conclusion. The generality of the method developed in this paper has been clearly exhibited in Part II, since all the solutions there obtained were determined from a single formula for the displacements, namely, formula (35). Moreover, the power of the method has been demonstrated by the diversity of the problems solved. These problems have been concerned with both complete and incomplete plates and with an extensive class of loads, namely, radial and axial mass force, uniform load, normal load proportional to $r^{2}$, concentrated normal load, and shearing traction proportional to $r$. Furthermore, with each type of load a large number of edge conditions have been studied.

Further evidence of both the power and generality of this method is seen in the ease and rigor with which it has been possible to find the values of the arbitrary constants $K_{1}, K_{3}$, and $C_{2}$ in the case of a complete plate. Certain writers, in attempting to determine these constants, have allowed themselves to be influenced by speculation concerning the physical nature of the problem. For instance, both de Saint-Venant and Love were led to give incorrect solutions in the problem of central load by assuming that $K_{3}=0$, an error due to the assumption that $w$ must be finite at the center of a complete plate.

Since the solutions given in this paper are of the two-dimensional type, a comparison with solutions of three-dimensional character is natural; there arises at once the question of relative accuracy. It has already been pointed out that Clemmow's experiments show that the best agreement with the actual deflection of a clamped plate is given by Case C-I, which is a twodimensional solution, and not by either Clemmow's three-dimensional solution or Nádai's. Clemmow has attributed this to a mere coincidence, since he (and Nádai also) has taken the stand that the correct solution for a plate can only be found from a three-dimensional solution, a two-dimensional solution being, of necessity, a less accurate approximation to the true physical situation. The position taken by Clemmow and Nádai would be a justifiable one if it were possible to give a correct mathematical definition of the boundary conditions at an edge and subsequently to find a three-dimensional solution in agreement with this mathematical definition. But Clemmow's experiments have clearly shown that it is not possible to give an accurate mathematical description of the boundary conditions at an edge, even for such a simple case as that of a clamped plate. 
Furthermore, there are only two types of boundary conditions which can be handled by Nádai's method; Clemmow is able to go beyond these two types, but only at the expense of extremely complicated computations. On the other hand, there seems to be no limit to the number of boundary conditions which can be studied by means of our two-dimensional method; moreover, the computations are much simplified when a two-dimensional solution is used. Finally, in practice, a two-dimensional solution is actually to be preferred to a three-dimensional one; for not only is the former simpler in structure, but, what is more, the results obtained from it may be truer physically (when a proper mathematical definition of the boundary conditions at the edge is used) than the results obtained from a three-dimensional solution whose boundary conditions do not so closely approximate those actually existing.

The application of our method is not limited to circular plates of constant thickness. The method may also be applied to circular plates of variable thickness, to rectangular plates of either constant or variable thickness, and, under the heading of one-dimensional problems, to moderately thin rods of either constant or variable thickness; in fact, this method may be used in any problem where Garabedian's method is applicable. So far as the author is aware no method other than Garabedian's has been developed which can be applied to such broad classes of problems. Moreover, the author is convinced that his method, or the closely parallel method of Garabedian, affords the most natural and satisfactory machinery for handling the two-dimensional problems of elasticity. They seem to be in every way superior, by virtue of their power, generality, and simplicity, to any two-dimensional method hitherto advanced.

University of Cincinnati, Cincinnati, OHio 